\title{
Selective Macrocycle Formation in Cavitands
}

Ji-Min Yang, ${ }^{1}$ Yang Yu, ${ }^{2}$ Julius Rebek Jr.*1

${ }^{1}$ Skaggs Institute for Chemical Biology and Department of Chemistry, The Scripps Research Institute, 10550 North Torrey Pines Road, La Jolla, CA 92037, USA

${ }^{2}$ Center for Supramolecular Chemistry \& Catalysis and Department of Chemistry, College of Science, Shanghai University, 99 Shang-Da Road, Shanghai 200444, China

e-mail: jrebek@ scripps.edu

\section{Table of Contents}

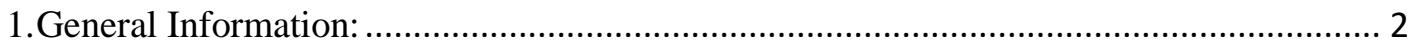

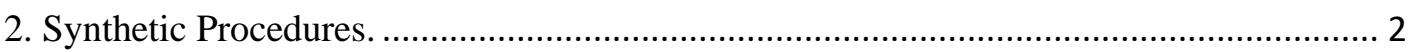

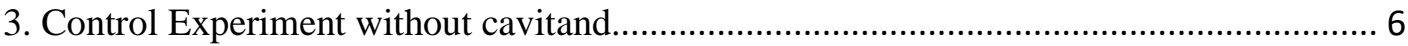

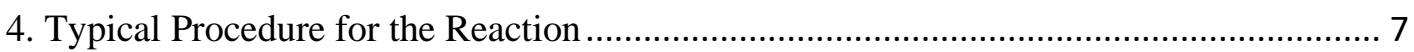

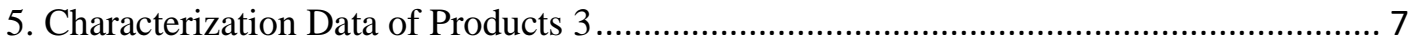

6. ${ }^{1} \mathrm{H}$ NMR Spectra and COSY NMR Spectra of 2a@cavitand 1 ....................................... 12

7. Typical Procedure for Monitoring the Reaction.......................................................... 14

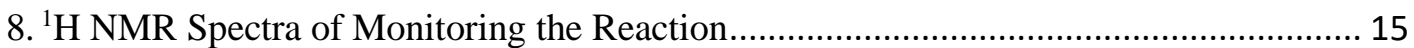

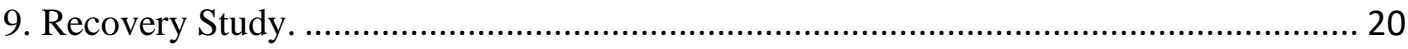

11. Procedure for the Allyl Deuterated Product. ................................................................ 23

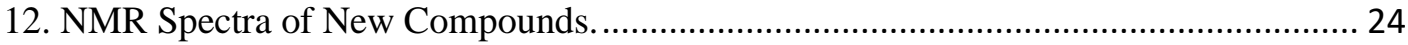

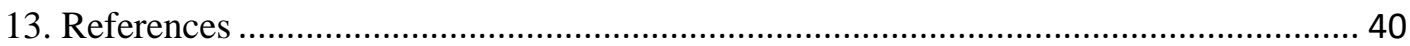




\section{General Information:}

Unless otherwise indicated, chemicals and solvents were used as received from TCI, Alfa Aesar, Combi-Blocks and Aldrich. Reactions were performed under a positive pressure of nitrogen, unless otherwise noted. ${ }^{1} \mathrm{H}$ and ${ }^{13} \mathrm{C}$ NMR spectra were obtained at $600 \mathrm{MHz}$ and $151 \mathrm{MHz}$, respectively, on a Bruker DRX-600 spectrometer equipped with a $5 \mathrm{~mm}$ DCH cryoprobe. Spectra were recorded at $298 \mathrm{~K}$ unless otherwise noted. NMR solvents were purchased from Cambridge Isotope Laboratories and Aldrich. Mass spectrometry was performed at the Scripps Center for Metabolomics and Mass Spectrometry.

\section{Synthetic Procedures.}

Cavitand $\mathbf{1}^{1}$ and dialdehydes $\mathbf{2 a - 2 f}$ were prepared from the corresponding diols according to previously reported procedures. ${ }^{2}$

Synthesis of dialdehydes 2a-2f.

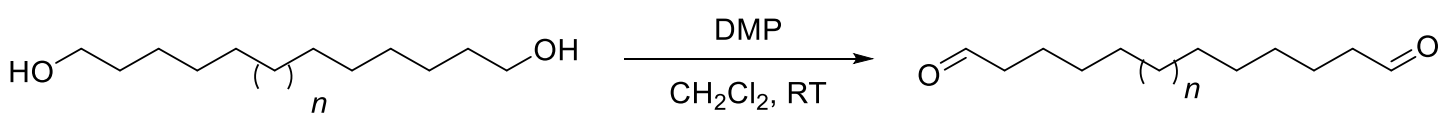

To a solution of diol $(1 \mathrm{mmol})$ in dry $\mathrm{CH}_{2} \mathrm{Cl}_{2}(100 \mathrm{~mL})$ was added Dess-Martin periodinane ( $1.26 \mathrm{~g}, 3 \mathrm{mmol}, 3$ equiv.) in portions at $0{ }^{\circ} \mathrm{C}$ and the reaction mixture was allowed to stir for $2 \mathrm{~h}$ at room temperature. It was then treated with saturated aq. solution of $\mathrm{Na}_{2} \mathrm{~S}_{2} \mathrm{O}_{3} \cdot 5 \mathrm{H}_{2} \mathrm{O}(10 \mathrm{~mL})$ and $\mathrm{NaHCO}_{3}(10 \mathrm{~mL})$ and stirred for $20 \mathrm{~min}$. The solution was extracted with $\mathrm{CH}_{2} \mathrm{Cl}_{2}(3 \times 20 \mathrm{~mL})$. The combined organic layers were washed with water, brine, dried $\left(\mathrm{Na}_{2} \mathrm{SO}_{4}\right)$ and concentrated under vacuum to afford the crude product. The resulting residue was purified by chromatography on silica gel (petroleum ether/ethyl acetate, $\mathrm{PE} / \mathrm{EA}=10: 1, \mathrm{v} / \mathrm{v}$ ) to afford the desired product.

2a, yield: 86\%, white solid. ${ }^{1} \mathrm{H}$ NMR $(600 \mathrm{MHz}$, Chloroform- $d$ ) $\delta 9.76(\mathrm{t}, J=1.9 \mathrm{~Hz}$, $2 \mathrm{H}), 2.42(\mathrm{td}, J=7.4,1.9 \mathrm{~Hz}, 4 \mathrm{H}), 1.65-1.59(\mathrm{~m}, 4 \mathrm{H}), 1.34-1.25(\mathrm{~m}, 12 \mathrm{H}) .{ }^{13} \mathrm{C} \mathrm{NMR}$ 
$\left(151 \mathrm{MHz}, \mathrm{CDCl}_{3}\right) \delta 203.06,44.03,29.43,29.42,29.25,22.18$. Spectral data is in agreement with literature. ${ }^{3}$

2b, yield: 88\%, white solid. ${ }^{1} \mathrm{H}$ NMR (600 MHz, Chloroform- $d$ ) $\delta 9.76(\mathrm{t}, J=1.9 \mathrm{~Hz}$, 2H), $2.42(\mathrm{td}, J=7.4,1.9 \mathrm{~Hz}, 4 \mathrm{H}), 1.65-1.57(\mathrm{~m}, 4 \mathrm{H}), 1.43-1.13(\mathrm{~m}, 14 \mathrm{H}) .{ }^{13} \mathrm{C}$ NMR $\left(151 \mathrm{MHz}, \mathrm{CDCl}_{3}\right) \delta 203.09,44.01,29.56,29.46,29.42,29.24,22.17$. Spectral data is in agreement with literature. ${ }^{4}$

2c, yield: 88\%, white solid. ${ }^{1} \mathrm{H}$ NMR (600 MHz, Chloroform- $d$ ) ${ }^{1} \mathrm{H}$ NMR (600 MHz, Chloroform- $d$ ) $\delta 9.76(\mathrm{t}, J=1.9 \mathrm{~Hz}, 2 \mathrm{H}), 2.42(\mathrm{td}, J=7.4,1.9 \mathrm{~Hz}, 4 \mathrm{H}), 1.65-1.57(\mathrm{~m}$, 4H), $1.37-1.23(\mathrm{~m}, 16 \mathrm{H}) .{ }^{13} \mathrm{C} \mathrm{NMR}\left(151 \mathrm{MHz}, \mathrm{CDCl}_{3}\right) \delta 203.07,44.01,29.60,29.48$, $29.43,29.24,22.17$. Spectral data is in agreement with literature. ${ }^{5}$

2d, yield: 85\%, white solid. ${ }^{1} \mathrm{H}$ NMR (600 MHz, Chloroform- $d$ ) ${ }^{1} \mathrm{H}$ NMR (600 MHz, Chloroform- $d$ ) $\delta 9.76(\mathrm{t}, J=1.9 \mathrm{~Hz}, 2 \mathrm{H}), 2.42(\mathrm{td}, J=7.4,1.9 \mathrm{~Hz}, 4 \mathrm{H}), 1.63(\mathrm{p}, J=7.3$ $\mathrm{Hz}, 4 \mathrm{H}), 1.36-1.21(\mathrm{~m}, 18 \mathrm{H}) .{ }^{13} \mathrm{C} \mathrm{NMR}\left(151 \mathrm{MHz}, \mathrm{CDCl}_{3}\right) \delta 203.15,44.05,29.70$, 29.67, 29.54, 29.48, 29.29, 22.21. HRMS (ESI): Calcd. for $\mathrm{C}_{15} \mathrm{H}_{29} \mathrm{O}_{2}[\mathrm{M}+\mathrm{H}]^{+}$241.2162, Found: 241.2173.

2e, yield: 89\%, white solid. ${ }^{1} \mathrm{H}$ NMR (600 MHz, Chloroform- $d$ ) $\delta 9.76(\mathrm{t}, J=1.9 \mathrm{~Hz}$, $2 \mathrm{H}), 2.42(\mathrm{td}, J=7.4,1.9 \mathrm{~Hz}, 4 \mathrm{H}), 1.68-1.58(\mathrm{~m}, 4 \mathrm{H}), 1.37-1.21(\mathrm{~m}, 20 \mathrm{H}) .{ }^{13} \mathrm{C}$ NMR $\left(151 \mathrm{MHz}, \mathrm{CDCl}_{3}\right) \delta 203.11,44.02,29.69,29.65,29.51,29.45,29.26,22.18$. Spectral data is in agreement with literature. ${ }^{6}$

2f, yield: 82\%, white solid. ${ }^{1} \mathrm{H}$ NMR (600 MHz, Chloroform- $d$ ) $\delta 9.76(\mathrm{t}, J=1.9 \mathrm{~Hz}$, $2 \mathrm{H}), 2.42(\mathrm{td}, J=7.4,1.9 \mathrm{~Hz}, 4 \mathrm{H}), 1.62(\mathrm{p}, J=7.4 \mathrm{~Hz}, 4 \mathrm{H}), 1.36-1.22(\mathrm{~m}, 24 \mathrm{H}) .{ }^{13} \mathrm{C}$ NMR (151 MHz, $\left.\mathrm{CDCl}_{3}\right) \delta 203.17,44.07,29.79,29.77,29.72,29.57,29.50,29.31$, 22.24. HRMS (ESI): Calcd. for $\mathrm{C}_{18} \mathrm{H}_{35} \mathrm{O}_{2}[\mathrm{M}+\mathrm{H}]^{+}$283.2632, Found: 283.2641. 


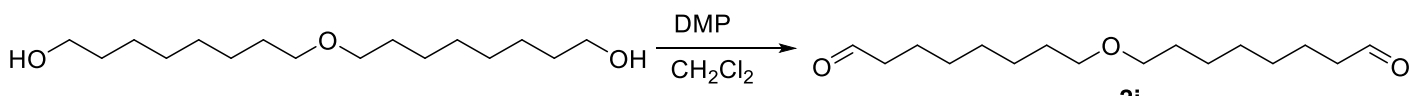

S2i was prepared according to previously reported procedures. ${ }^{7}$ To a solution of diol (274 mg, $1 \mathrm{mmol})$ in dry $\mathrm{CH}_{2} \mathrm{Cl}_{2}(100 \mathrm{~mL})$ was added Dess-Martin periodinane (1.26 g, $3 \mathrm{mmol}, 3$ equiv.) in portions at $0{ }^{\circ} \mathrm{C}$ and the reaction mixture was allowed to stir for $2 \mathrm{~h}$ at room temperature. It was then treated with saturated aq. solution of $\mathrm{Na}_{2} \mathrm{~S}_{2} \mathrm{O}_{3} \cdot 5 \mathrm{H}_{2} \mathrm{O}(10 \mathrm{~mL})$ and $\mathrm{NaHCO}_{3}(10 \mathrm{~mL})$ and stirred for $20 \mathrm{~min}$. The solution was extracted with $\mathrm{CH}_{2} \mathrm{Cl}_{2}(3 \times 20 \mathrm{~mL})$. The combined organic layers were washed with water, brine, dried $\left(\mathrm{Na}_{2} \mathrm{SO}_{4}\right)$ and concentrated under vacuum to afford the crude product. The resulting residue was purified by chromatography on silica gel (petroleum ether/ethyl acetate, $\mathrm{PE} / \mathrm{EA}=10: 1, \mathrm{v} / \mathrm{v}$ ) to afford the desired product $\mathbf{2} \mathbf{i}$ as color less oil in $85 \%$ yield. ${ }^{1} \mathrm{H}$ NMR $(600 \mathrm{MHz}$, Chloroform- $d$ ) $\delta 9.75(\mathrm{t}, J=1.8 \mathrm{~Hz}, 2 \mathrm{H}), 3.38(\mathrm{t}, J$ $=6.7 \mathrm{~Hz}, 4 \mathrm{H}), 2.41(\mathrm{td}, J=7.3,1.9 \mathrm{~Hz}, 4 \mathrm{H}), 1.67-1.51(\mathrm{~m}, 9 \mathrm{H}), 1.37-1.30(\mathrm{~m}, 12 \mathrm{H})$.

${ }^{13} \mathrm{C} \mathrm{NMR}\left(151 \mathrm{MHz}, \mathrm{CDCl}_{3}\right) \delta 202.99,70.95,43.99,29.78,29.30,29.20,26.10,22.11$. HRMS (ESI): Calcd. for $\mathrm{C}_{16} \mathrm{H}_{31} \mathrm{O}_{3}[\mathrm{M}+\mathrm{H}]^{+}$271.2268, Found: 271.2270.

2g, yield: 86\%, colorless oil. ${ }^{1} \mathrm{H}$ NMR $(600 \mathrm{MHz}$, Chloroform- $d$ ) $\delta 9.76(\mathrm{t}, J=1.8 \mathrm{~Hz}$, 2H), $3.39(\mathrm{t}, J=6.5 \mathrm{~Hz}, 4 \mathrm{H}), 2.44(\mathrm{td}, J=7.4,1.8 \mathrm{~Hz}, 4 \mathrm{H}), 1.67-1.56(\mathrm{~m}, 8 \mathrm{H}), 1.44$ - 1.35 (m, 4H). ${ }^{13} \mathrm{C}$ NMR (151 MHz, $\left.\mathrm{CDCl}_{3}\right) \delta 202.80,70.68,43.95,25.95,22.01$, 22.01. HRMS (ESI): Calcd. for $\mathrm{C}_{12} \mathrm{H}_{23} \mathrm{O}_{3}[\mathrm{M}+\mathrm{H}]^{+}$215.1642, Found: 215.1649.

\section{Synthesis of dialdehydes $2 \mathrm{~h}$ and $2 \mathrm{j}$.}

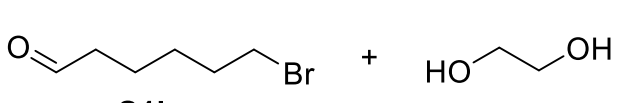

S1h

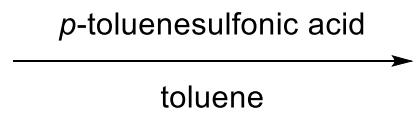

toluene

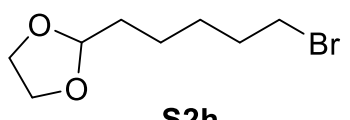

$\mathrm{s} 2 \mathrm{~h}$

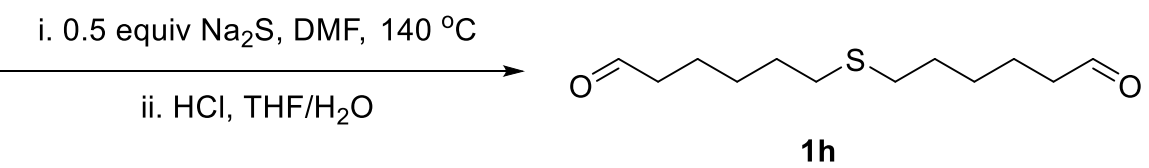

To a solution of 6-bromohexanal $\mathbf{S 1 h}(895 \mathrm{mg}, 5 \mathrm{mmol})$ and ethylene glycol (341 mg, $5.5 \mathrm{mmol})$ in toluene $(20 \mathrm{~mL})$ was added $p$-toluenesulfonic acid monohydrate $(95 \mathrm{mg}$, 
$0.5 \mathrm{mmol}$ ). The mixture was heated to $110^{\circ} \mathrm{C}$. After 2 hours, the reaction was cooled to room temperature and diluted with $50 \mathrm{~mL}$ of ethyl acetate. This solution was washed with $2 \times 50 \mathrm{~mL}$ of aqueous $\mathrm{NaHCO}_{3}$ saturated solution and dried over $\mathrm{Na}_{2} \mathrm{SO}_{4}$ and concentrated under vacuum to afford the crude $\mathbf{S} \mathbf{2 h}$ in quantitative yield. The crude $\mathbf{S} \mathbf{2 h}$ was taken for the next step without further purification.

To a solution of $\mathbf{S 2 h}$ ( $5 \mathrm{mmol})$ in dry DMF $(5 \mathrm{~mL})$ was added sodium sulfide (195 mg, $2.5 \mathrm{mmol}$ ) in one portion under $\mathrm{N}_{2}$ atmosphere and the reaction mixture was allowed to stir for overnight under $140{ }^{\circ} \mathrm{C}$. Then the reaction was cooled to room temperature and diluted with $\mathrm{H}_{2} \mathrm{O}(30 \mathrm{~mL})$. The solution was extracted with EA $(3 \times 30 \mathrm{~mL})$ and dried over $\mathrm{Na}_{2} \mathrm{SO}_{4}$ and concentrated under vacuum to afford a yellow oil. The oil was treated with a solution of THF $(50 \mathrm{~mL})$ and $20 \%$ aq $\mathrm{HCl}(50 \mathrm{~mL})$, and the mixture was stirred for $24 \mathrm{~h}$ at $37{ }^{\circ} \mathrm{C}$. Then aq $\mathrm{NaHCO}_{3}(400 \mathrm{~mL})$ was added slowly and the mixture was extracted with $\mathrm{Et}_{2} \mathrm{O}(3 \times 100 \mathrm{~mL})$. The organic layers were combined, dried $\left(\mathrm{Na}_{2} \mathrm{SO}_{4}\right)$, filtered, and concentrated under vacuum. The residue was purified by flash chromatography to afford $\mathbf{2 h}$ as a colorless oil, 62\% yield for 3 steps. ${ }^{1} \mathrm{H}$ NMR (600 MHz, Chloroform- $d$ ) $\delta 9.75(\mathrm{t}, J=1.7 \mathrm{~Hz}, 2 \mathrm{H}), 2.52-2.47(\mathrm{~m}, 4 \mathrm{H}), 2.43(\mathrm{td}, J=7.3$, $1.7 \mathrm{~Hz}, 4 \mathrm{H}), 1.66-1.55(\mathrm{~m}, 8 \mathrm{H}), 1.46-1.38(\mathrm{~m}, 4 \mathrm{H}) .{ }^{13} \mathrm{C} \mathrm{NMR}\left(151 \mathrm{MHz}, \mathrm{CDCl}_{3}\right) \delta$ 202.63, 43.83, 31.99, 29.44, 28.42, 21.73. HRMS (ESI): Calcd. for $\mathrm{C}_{12} \mathrm{H}_{23} \mathrm{O}_{2} \mathrm{~S}[\mathrm{M}+\mathrm{H}]^{+}$ 231.1413, Found: 231.1423

2j, 66\% yield for 3 steps, colorless oil. ${ }^{1} \mathrm{H}$ NMR $(600 \mathrm{MHz}$, Chloroform- $d$ ) $\delta 9.76(\mathrm{t}, J$ $=1.8 \mathrm{~Hz}, 2 \mathrm{H}), 2.53-2.46(\mathrm{~m}, 4 \mathrm{H}), 2.44-2.39(\mathrm{~m}, 4 \mathrm{H}), 1.63-1.54(\mathrm{~m}, 8 \mathrm{H}), 1.40-$ $1.29(\mathrm{~m}, 12 \mathrm{H}) .{ }^{13} \mathrm{C} \mathrm{NMR}\left(151 \mathrm{MHz}, \mathrm{CDCl}_{3}\right) \delta 202.94,43.97,32.24,29.69,29.13,29.07$, 28.77, 22.09. HRMS (ESI): Calcd. for $\mathrm{C}_{16} \mathrm{H}_{31} \mathrm{O}_{2} \mathrm{~S}[\mathrm{M}+\mathrm{H}]^{+}$287.2039, Found: 287.2049 


\section{Control Experiments without cavitand.}

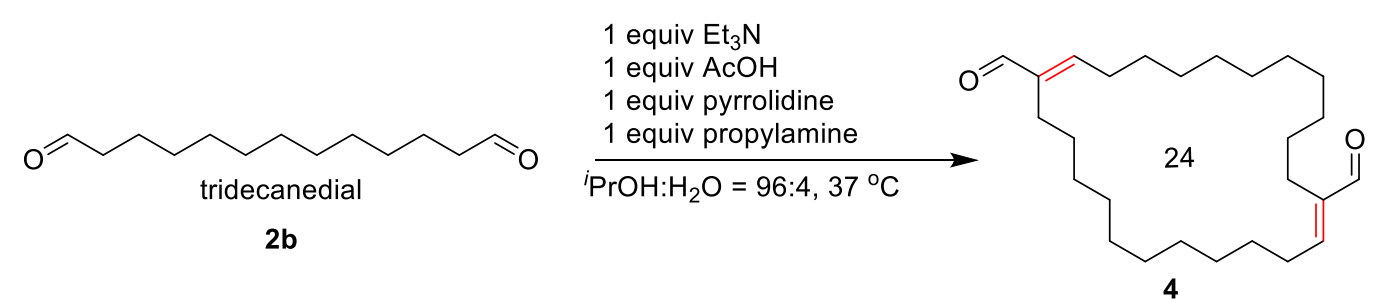

Under a $\mathrm{N}_{2}$ atmosphere, tridencanedial $2 \mathbf{b}(8.5 \mathrm{mg}, 0.04 \mathrm{mmol}), \mathrm{H}_{2} \mathrm{O}(0.8 \mathrm{~mL})$ and ${ }^{i} \mathrm{PrOH}(20 \mathrm{~mL})$ were introduced into a round bottom flask. Then $\mathrm{Et}_{3} \mathrm{~N}(4 \mathrm{mg}, 0.04$ $\mathrm{mmol}), \mathrm{AcOH}$ (2.4 mg, $0.04 \mathrm{mmol})$, pyrrolidine $(2.8 \mathrm{mg}, 0.04 \mathrm{mmol})$, and propylamine ( $2.9 \mathrm{mg}, 0.04 \mathrm{mmol}$ ) were injected into the flask. The resulting mixture was stirred at $37^{\circ} \mathrm{C}$ for another $20 \mathrm{~h}$, then concentrated in vacuo and the residue was purified by flash column chromatography $(\mathrm{PE} / \mathrm{EA}=20: 1, \mathrm{v} / \mathrm{v})$. The conversion of this reaction is $52 \%$ based the recovery of $\mathbf{2 b}$ and the main product $\mathbf{4}$ obtained in $20 \%$ yield as a white solid. ${ }^{1} \mathrm{H}$ NMR (600 MHz, Chloroform- $d$ ) $\delta 9.36(\mathrm{~s}, 2 \mathrm{H}), 6.47-6.40(\mathrm{~m}, 2 \mathrm{H}), 2.38-2.32(\mathrm{~m}$, $4 \mathrm{H}), 2.27-2.20(\mathrm{~m}, 4 \mathrm{H}), 1.55-1.47(\mathrm{~m}, 4 \mathrm{H}), 1.39-1.24(\mathrm{~m}, 28 \mathrm{H}) .{ }^{13} \mathrm{C}$ NMR $(151$ $\left.\mathrm{MHz}, \mathrm{CDCl}_{3}\right) \delta 195.60,195.53,155.50,155.47,143.90,143.79,29.66,29.58,29.55$, $29.46,29.41,29.31,29.15,29.09,29.05,28.99,28.96,28.80,28.71,28.67,28.55,28.51$, 28.31, 23.93, 23.88. HRMS (ESI): Calcd. for $\mathrm{C}_{26} \mathrm{H}_{45} \mathrm{O}_{2}[\mathrm{M}+\mathrm{H}]^{+}$389.3414, Found: 389.3421 .

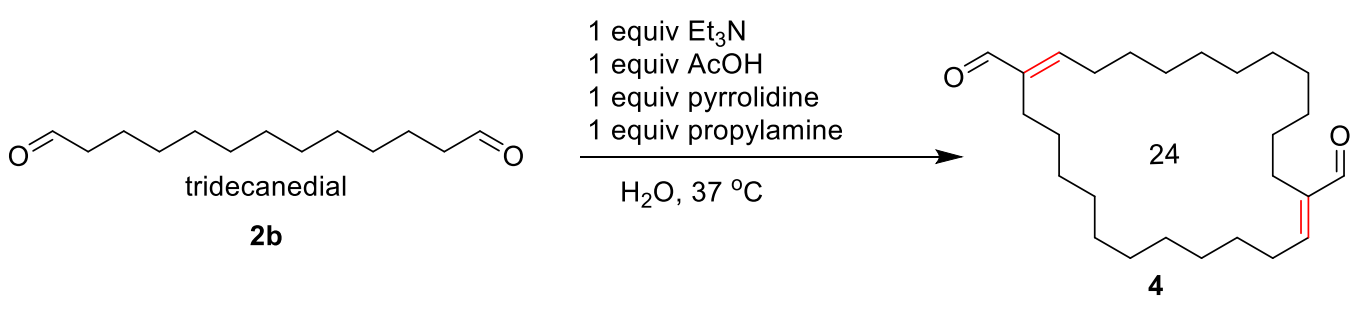

Under a $\mathrm{N}_{2}$ atmosphere, tridencanedial $2 \mathbf{b}(8.5 \mathrm{mg}, 0.04 \mathrm{mmol})$ and $\mathrm{H}_{2} \mathrm{O}(20 \mathrm{~mL})$ were introduced into a round bottom flask. Then $\mathrm{Et}_{3} \mathrm{~N}$ ( $\left.4 \mathrm{mg}, 0.04 \mathrm{mmol}\right), \mathrm{AcOH}$ (2.4 mg, $0.04 \mathrm{mmol})$, pyrrolidine $(2.8 \mathrm{mg}, 0.04 \mathrm{mmol})$, and propylamine $(2.9 \mathrm{mg}, 0.04 \mathrm{mmol})$ 
were injected into the flask. The resulting mixture was stirred at $37^{\circ} \mathrm{C}$ for another $20 \mathrm{~h}$, then extracted with $\mathrm{CHCl}_{3}(20 \mathrm{~mL} \times 3)$. The combined organic phases were washed with brine and dried over $\mathrm{Na}_{2} \mathrm{SO}_{4}$. The filtrate was concentrated in vacuo and the residue was analyzed by ${ }^{1} \mathrm{H}$ NMR using mesitylene as the internal standard.

\section{Typical Procedure for the Chaperoned Reaction}

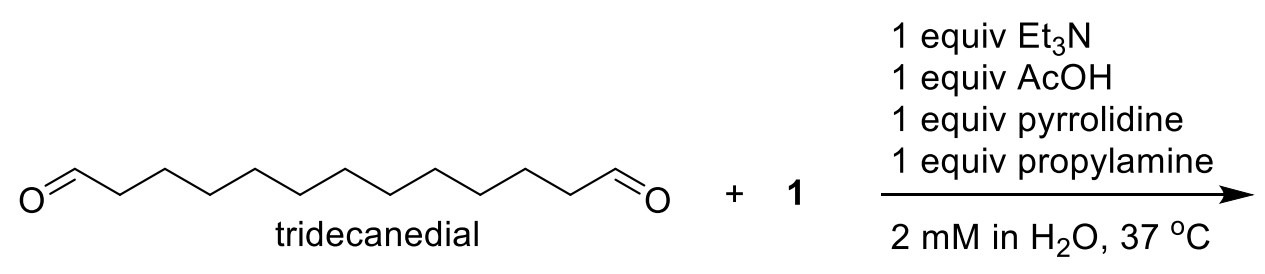

2b

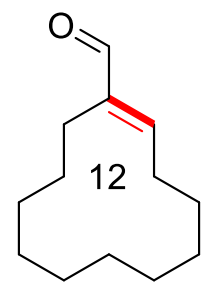

$3 b$

Under a $\mathrm{N}_{2}$ atmosphere, tridencanedial $\mathbf{2 b}(8.5 \mathrm{mg}, 0.04 \mathrm{mmol})$ and $\mathbf{1}(84 \mathrm{mg}, 0.05$ mmol) were introduced into a round bottom flask. Then $20 \mathrm{~mL} \mathrm{H}_{2} \mathrm{O}$ was injected into the flask in one portion. After stirring for $2 \mathrm{~h}$ at $37{ }^{\circ} \mathrm{C}, \mathrm{Et}_{3} \mathrm{~N}(4 \mathrm{mg}, 0.04 \mathrm{mmol}), \mathrm{AcOH}$ (2.4 mg, $0.04 \mathrm{mmol})$, pyrrolidine $(2.8 \mathrm{mg}, 0.04 \mathrm{mmol})$, and propylamine $(2.9 \mathrm{mg}, 0.04$ mmol) were injected into the flask. The resulting mixture was stirred at $37{ }^{\circ} \mathrm{C}$ for another $20 \mathrm{~h}$, then extracted with $\mathrm{CHCl}_{3}(20 \mathrm{~mL} \times 3)$. The combined organic phases were washed with brine and dried over $\mathrm{Na}_{2} \mathrm{SO}_{4}$. The filtrate was concentrated in vacuo and the residue was purified by flash column chromatography (PE/EA = 20:1, v/v).

\section{Characterization Data for Products 3}

(E)-cyclododec-1-ene-1-carbaldehyde $\mathbf{3 b}$<smiles>CCCCCCCCCCCC/C=C(/C=O)CC</smiles> 
${ }^{1} \mathrm{H}$ NMR (600 MHz, Chloroform- $d$ ) $\delta 9.38(\mathrm{~s}, 1 \mathrm{H}), 6.48(\mathrm{t}, J=8.1 \mathrm{~Hz}, 1 \mathrm{H}), 2.42-2.35$ $(\mathrm{m}, 2 \mathrm{H}), 2.31(\mathrm{t}, J=6.8 \mathrm{~Hz}, 2 \mathrm{H}), 1.66-1.60(\mathrm{~m}, 2 \mathrm{H}), 1.59-1.54(\mathrm{~m}, 2 \mathrm{H}), 1.46-1.32$ (m, 10H), $1.22-1.16(\mathrm{~m}, 2 \mathrm{H}) .{ }^{13} \mathrm{C}$ NMR (151 MHz, $\left.\mathrm{CDCl}_{3}\right) \delta 196.13,155.96,143.55$, $26.37,26.00,25.85,25.31,25.22,24.93,23.59,22.98,22.17,21.59$. Spectral data is in agreement with literature. ${ }^{8}$

(E)-cyclotridec-1-ene-1-carbaldehyde 3c

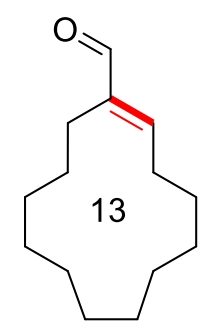

Colorless oil. ${ }^{1} \mathrm{H}$ NMR (600 MHz, Chloroform- $d$ ) $\delta 9.39$ (s, 1H), 6.44 (t, $J=7.4 \mathrm{~Hz}$, $1 \mathrm{H}), 2.45-2.40(\mathrm{~m}, 2 \mathrm{H}) 2.31(\mathrm{t}, J=7.1 \mathrm{~Hz}, 2 \mathrm{H}), 1.69-4.61(\mathrm{~m}, 2 \mathrm{H}), 1.54-1.47(\mathrm{~m}$, 2H), $1.39-1.20(\mathrm{~m}, 14 \mathrm{H}) .{ }^{13} \mathrm{C} \mathrm{NMR}\left(151 \mathrm{MHz}, \mathrm{CDCl}_{3}\right) \delta$ 195.62, 156.85, 144.36, 27.21, 27.13, 27.08, 26.47, 26.21, 25.98, 25.72, 25.43, 24.30, 22.62. HRMS (ESI): Calcd. for $\mathrm{C}_{14} \mathrm{H}_{24} \mathrm{O}[\mathrm{M}+\mathrm{H}]^{+}$209.1900, Found: 209.1896.

(E)-cyclotetradec-1-ene-1-carbaldehyde 3d

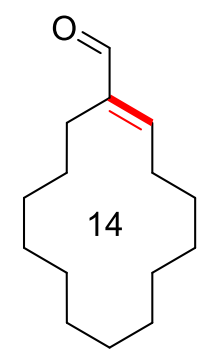

Colorless oil. ${ }^{1} \mathrm{H}$ NMR (600 MHz, Chloroform- $d$ ) $\delta 9.37$ (s, 1H), 6.45 (t, $J=8.0 \mathrm{~Hz}$, $1 \mathrm{H}), 2.38-2.32(\mathrm{~m}, 2 \mathrm{H}), 2.29-2.16(\mathrm{~m}, 2 \mathrm{H}), 1.57-1.52(\mathrm{~m}, 2 \mathrm{H}), 1.47-1.25(\mathrm{~m}$, 18H). ${ }^{13} \mathrm{C}$ NMR (151 MHz, $\left.\mathrm{CDCl}_{3}\right) \delta 195.69,155.27,144.41,27.86,27.55,27.02$, 26.90, 26.44, 26.21, 25.69, 24.90, 24.45, 24.15, 23.86, 23.70. HRMS (ESI): Calcd. for $\mathrm{C}_{15} \mathrm{H}_{27} \mathrm{O}[\mathrm{M}+\mathrm{H}]^{+}$223.2056, Found: 223.2062.

(E)-cyclopentadec-1-ene-1-carbaldehyde 3e 


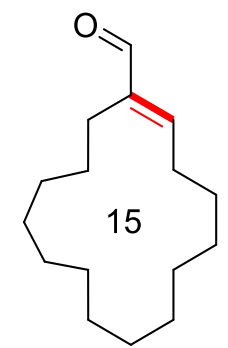

Colorless oil. ${ }^{1} \mathrm{H}$ NMR (600 MHz, Chloroform- $d$ ) $\delta 9.37$ (s, 1H), 6.47 (t, $J=7.7 \mathrm{~Hz}$, 1H), $2.38-2.32(\mathrm{~m}, 2 \mathrm{H}), 2.27-2.20(\mathrm{~m}, 2 \mathrm{H}), 1.56-1.50(\mathrm{~m}, 2 \mathrm{H}), 1.49-1.42(\mathrm{~m}$, 2H), $1.38-1.25(\mathrm{~m}, 18 \mathrm{H}) .{ }^{13} \mathrm{C}$ NMR $\left(151 \mathrm{MHz}, \mathrm{CDCl}_{3}\right) \delta 195.65,155.69,143.95$, 28.61, 27.58, 27.52, 26.90, 26.35, 26.24, 25.88, 23.74. HRMS (ESI): Calcd. for $\mathrm{C}_{16} \mathrm{H}_{29} \mathrm{O}[\mathrm{M}+\mathrm{H}]^{+}$237.2213, Found: 237.2219. Spectral data is in agreement with literature. $^{8}$

(E)-cycloheptadec-1-ene-1-carbaldehyde $\mathbf{3 f}$

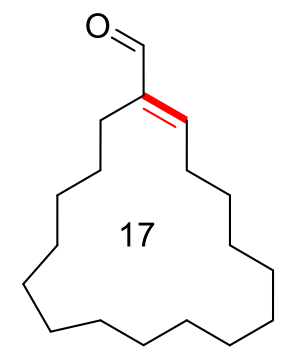

Colorless oil. ${ }^{1} \mathrm{H}$ NMR (600 MHz, Chloroform- $d$ ) $\delta 9.36(\mathrm{~s}, 1 \mathrm{H}), 6.44(\mathrm{t}, J=7.4 \mathrm{~Hz}$, $1 \mathrm{H}), 2.40-2.33(\mathrm{~m}, 2 \mathrm{H}), 2.27-2.21(\mathrm{~m}, 2 \mathrm{H}), 1.56-1.50(\mathrm{~m}, 2 \mathrm{H}), 1.41-1.27(\mathrm{~m}$, 24H). ${ }^{13} \mathrm{C}$ NMR (151 MHz, $\left.\mathrm{CDCl}_{3}\right) \delta 195.58,155.79,143.95,29.85,28.73,28.53$, $28.43,28.12,27.72,27.55,27.52,27.46,27.34,27.26,26.97,26.71,23.88$. HRMS (ESI): Calcd. for $\mathrm{C}_{18} \mathrm{H}_{33} \mathrm{O}[\mathrm{M}+\mathrm{H}]^{+}$265.2526, Found: 265.2542.

(E)-oxacyclododec-6-ene-6-carbaldehyde 3g<smiles>O=C/C1=C/CCCCCOCCCC1</smiles> 
Colorless oil. ${ }^{1} \mathrm{H}$ NMR (600 MHz, Chloroform- $d$ ) $\delta 9.38$ (s, 1H), 6.53 (t, $J=8.3 \mathrm{~Hz}$, $1 \mathrm{H}), 3.54-3.50(\mathrm{~m}, 2 \mathrm{H}), 3.49(\mathrm{t}, J=5.2 \mathrm{~Hz}, 2 \mathrm{H}), 2.40(\mathrm{q}, J=7.9 \mathrm{~Hz}, 2 \mathrm{H}), 2.34-2.29$ (m, 2H), $1.69-1.57$ (m, 8H). $\left.{ }^{13} \mathrm{C} \mathrm{NMR} \mathrm{(151} \mathrm{MHz,} \mathrm{CDCl}_{3}\right) \delta 195.93,154.71,145.71$, 71.24, 68.20, 27.95, 27.31, 27.05, 26.33, 26.17, 25.55, 22.57. HRMS (ESI): Calcd. for $\mathrm{C}_{12} \mathrm{H}_{21} \mathrm{O}_{2}[\mathrm{M}+\mathrm{H}]^{+}$197.1536, Found: 197.1537.

(E)-thiacyclododec-6-ene-6-carbaldehyde $\mathbf{3 h}$<smiles>CCCCCCSCCCCCC=C(C=O)C=O</smiles>

Colorless oil. ${ }^{1} \mathrm{H}$ NMR $(600 \mathrm{MHz}$, Chloroform- $d$ ) $\delta 9.39$ (s, 1H), 6.49 (t, $J=8.2 \mathrm{~Hz}$, $1 \mathrm{H}), 2.57(\mathrm{t}, J=7.2 \mathrm{~Hz}, 2 \mathrm{H}), 2.51(\mathrm{t}, J=6.7 \mathrm{~Hz}, 2 \mathrm{H}), 2.45-2.40(\mathrm{~m}, 2 \mathrm{H}), 2.30(\mathrm{t}, J=$ $7.1 \mathrm{~Hz}, 2 \mathrm{H}), 1.80-1.63(\mathrm{~m}, 6 \mathrm{H}), 1.58-1.49(\mathrm{~m}, 4 \mathrm{H}) .{ }^{13} \mathrm{C} \mathrm{NMR}\left(151 \mathrm{MHz}, \mathrm{CDCl}_{3}\right) \delta$ 195.73, 155.74, 143.49, 29.30, 28.19, 27.05, 27.03, 26.03, 25.64, 25.50, 25.30, 21.97. HRMS (ESI): Calcd. for $\mathrm{C}_{12} \mathrm{H}_{21} \mathrm{OS}[\mathrm{M}+\mathrm{H}]^{+}$213.1308, Found: 213.1315.

(E)-oxacyclohexadec-8-ene-8-carbaldehyde 3i

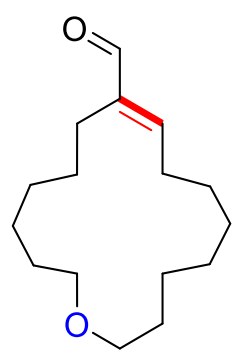

Colorless oil. ${ }^{1} \mathrm{H}$ NMR (600 MHz, Chloroform- $d$ ) $\delta 9.37$ (s, 1H), 6.49 (t, $J=7.9 \mathrm{~Hz}$, 1H), $3.48-3.44(\mathrm{~m}, 2 \mathrm{H}), 3.41(\mathrm{t}, J=5.6 \mathrm{~Hz}, 2 \mathrm{H}), 2.35(\mathrm{q}, J=7.9 \mathrm{~Hz}, 2 \mathrm{H}), 2.28-2.22$ $(\mathrm{m}, 2 \mathrm{H}), 1.62-1.38(\mathrm{~m}, 16 \mathrm{H}), 1.37-1.29(\mathrm{~m}, 2 \mathrm{H}) .{ }^{13} \mathrm{C} \mathrm{NMR}\left(151 \mathrm{MHz}, \mathrm{CDCl}_{3}\right) \delta$ 195.69, 155.28, 144.29, 70.40, 69.56, 29.05, 28.97, 28.90, 28.54, 28.37, 28.07, 27.60, 27.29, 26.09, 26.04, 23.57. Colorless oil. HRMS (ESI): Calcd. for $\mathrm{C}_{16} \mathrm{H}_{29} \mathrm{O}_{2}[\mathrm{M}+\mathrm{H}]^{+}$ 253.2162, Found: 253.2169. 
(E)-thiacyclohexadec-8-ene-8-carbaldehyde $\mathbf{3 j}$

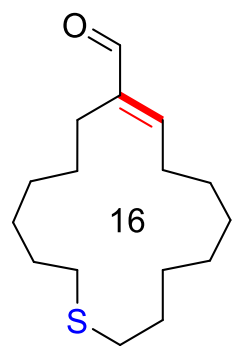

Colorless oil. ${ }^{1} \mathrm{H}$ NMR (600 MHz, Chloroform- $d$ ) $\delta 9.38$ (s, 1H), 6.48 (t, $J=7.9 \mathrm{~Hz}$, $1 \mathrm{H}), 2.51(\mathrm{t}, J=7.1 \mathrm{~Hz}, 2 \mathrm{H}), 2.48-2.45(\mathrm{~m}, 2 \mathrm{H}), 2.38(\mathrm{q}, J=7.8 \mathrm{~Hz}, 2 \mathrm{H}), 2.27(\mathrm{t}, J=$ $7.5 \mathrm{~Hz}, 2 \mathrm{H}), 1.61-1.56(\mathrm{~m}, 4 \mathrm{H}), 1.54-1.50(\mathrm{~m}, 2 \mathrm{H}), 1.48-1.37(\mathrm{~m}, 10 \mathrm{H}), 1.34-$ $1.28(\mathrm{~m}, 2 \mathrm{H}) .{ }^{13} \mathrm{C}$ NMR $\left(151 \mathrm{MHz}, \mathrm{CDCl}_{3}\right) \delta 195.63,155.44,143.80,30.83,30.06$, 29.23, 29.01, 28.14, 28.00, 27.83, 27.76, 27.33, 26.64, 23.96. Colorless oil. HRMS (ESI): Calcd. for $\mathrm{C}_{16} \mathrm{H}_{29} \mathrm{OS}[\mathrm{M}+\mathrm{H}]^{+}$269.1934, Found: 269.1942 . 


\section{6. ${ }^{1} \mathrm{H}$ NMR Spectra and COSY NMR Spectra of 2a@cavitand 1}

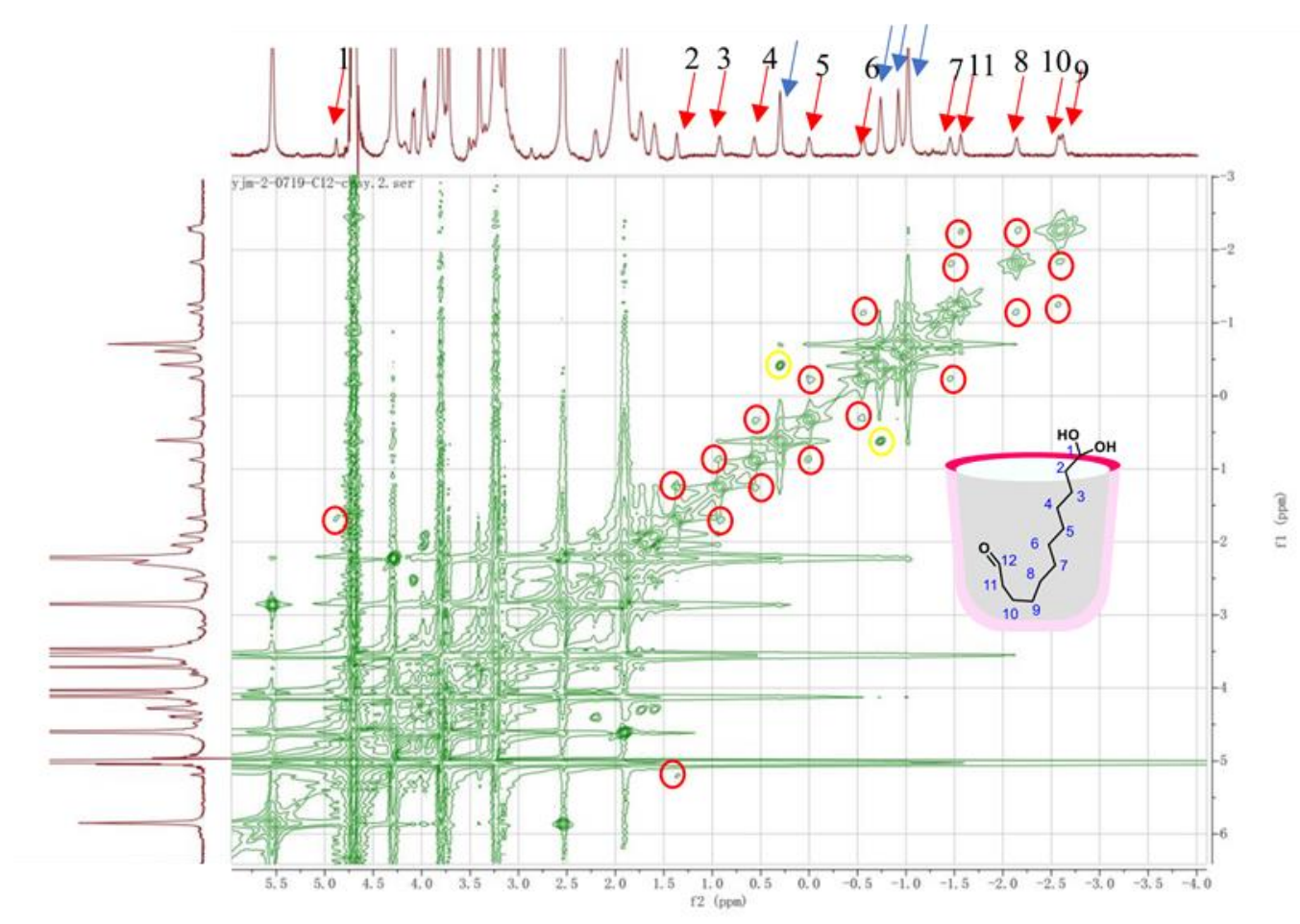

Figure S1. Upfield portions of the COSY NMR spectrum (600 MHz, 298 K, D 2 O) of 2a@cavitand 1 (4.0 mM). Peaks labeled with blue arrows for complex A, peaks labeled with red arrows for complex $\mathbf{B}$. 


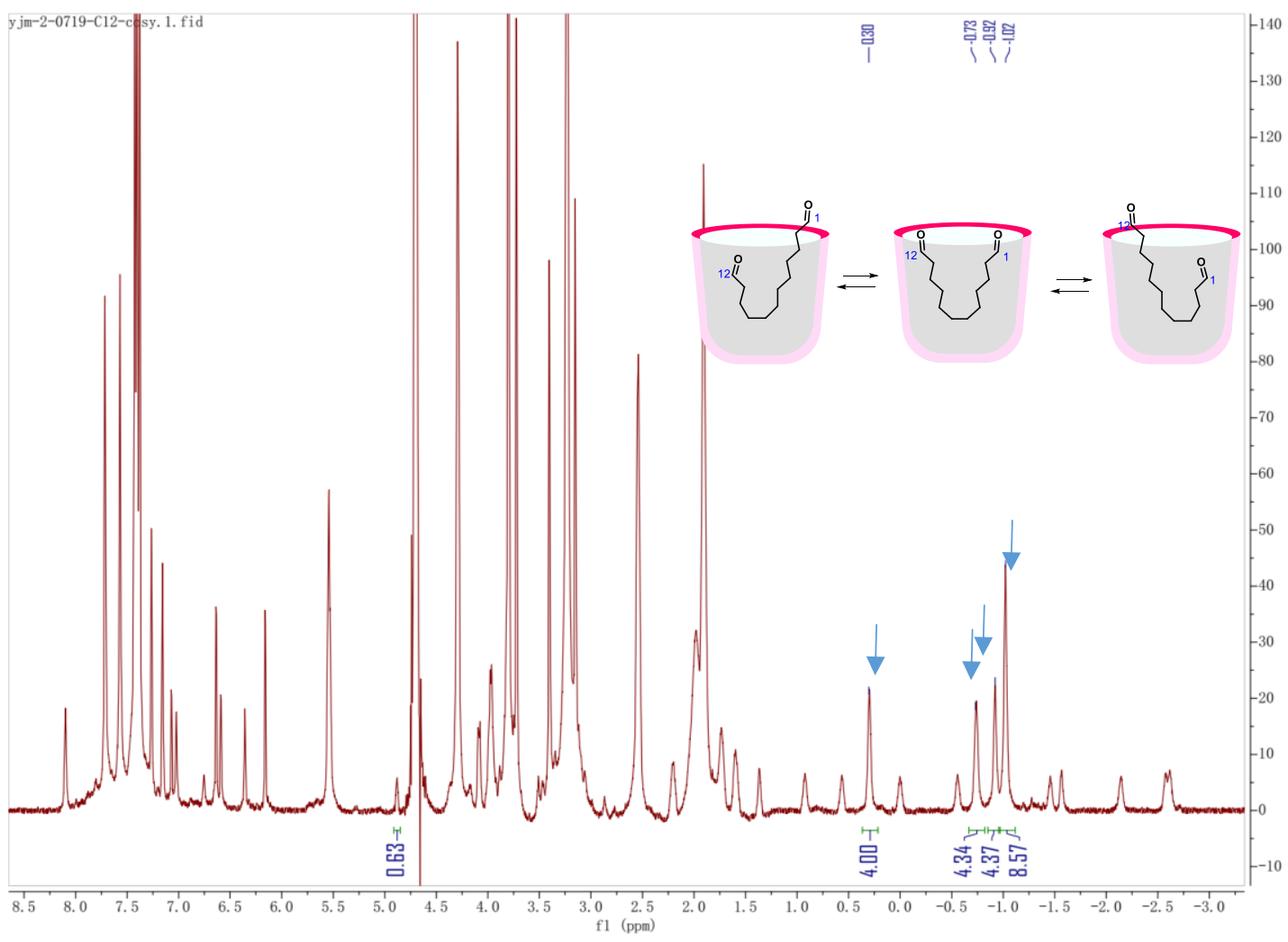

Figure S2. ${ }^{1} \mathrm{H}$ NMR spectra of complex A (blue arrows, $4.0 \mathrm{mM}$ ).

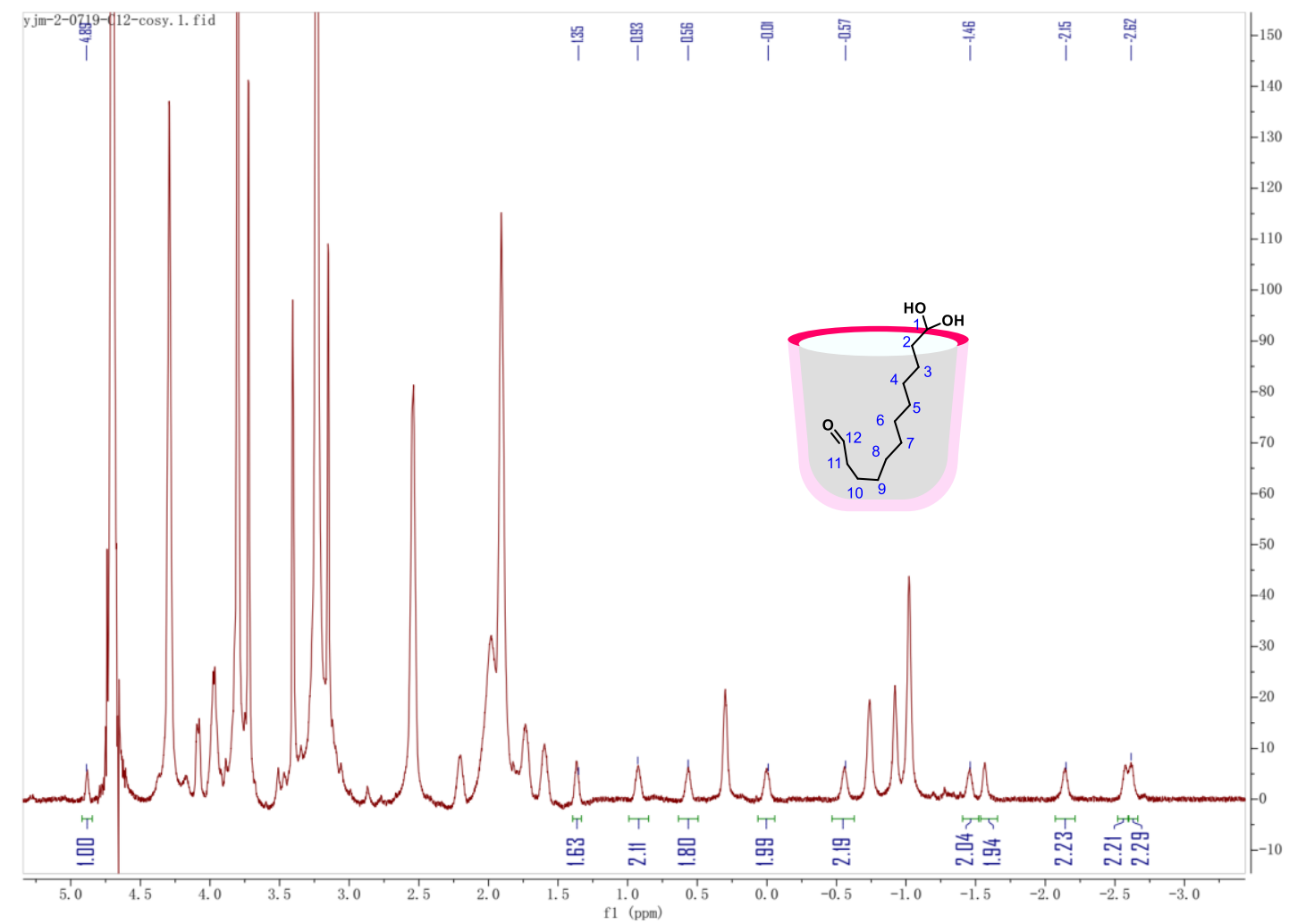

Figure S3. ${ }^{1} \mathrm{H}$ NMR spectra of complex B $(4.0 \mathrm{mM})$. 


\section{Typical Procedure for Monitoring the Reaction}

A solution of compound $2\left(50 \mu \mathrm{L}, 20 \mathrm{mM}\right.$ in $\left.\mathrm{CDCl}_{3}\right)$ was introduced to an NMR tube. After evaporated the solvent, 1 equivalent of 1 in $0.5 \mathrm{~mL} \mathrm{D}_{2} \mathrm{O}$ was injected and sonicated for $2 \mathrm{~h}$ at $37^{\circ} \mathrm{C}$. Then 1 equivalent each of $\mathrm{Et}_{3} \mathrm{~N}, \mathrm{AcOH}$, pyrrolidine and propylamine $\left(100 \mathrm{mM}\right.$ solution in $\left.\mathrm{D}_{2} \mathrm{O}\right)$ were injected into the NMR tube, then sonicated at $37^{\circ} \mathrm{C}$. The reaction was monitored by ${ }^{1} \mathrm{H}$ NMR. 


\section{8. ${ }^{1} \mathrm{H}$ NMR Spectra while Monitoring the Reaction}

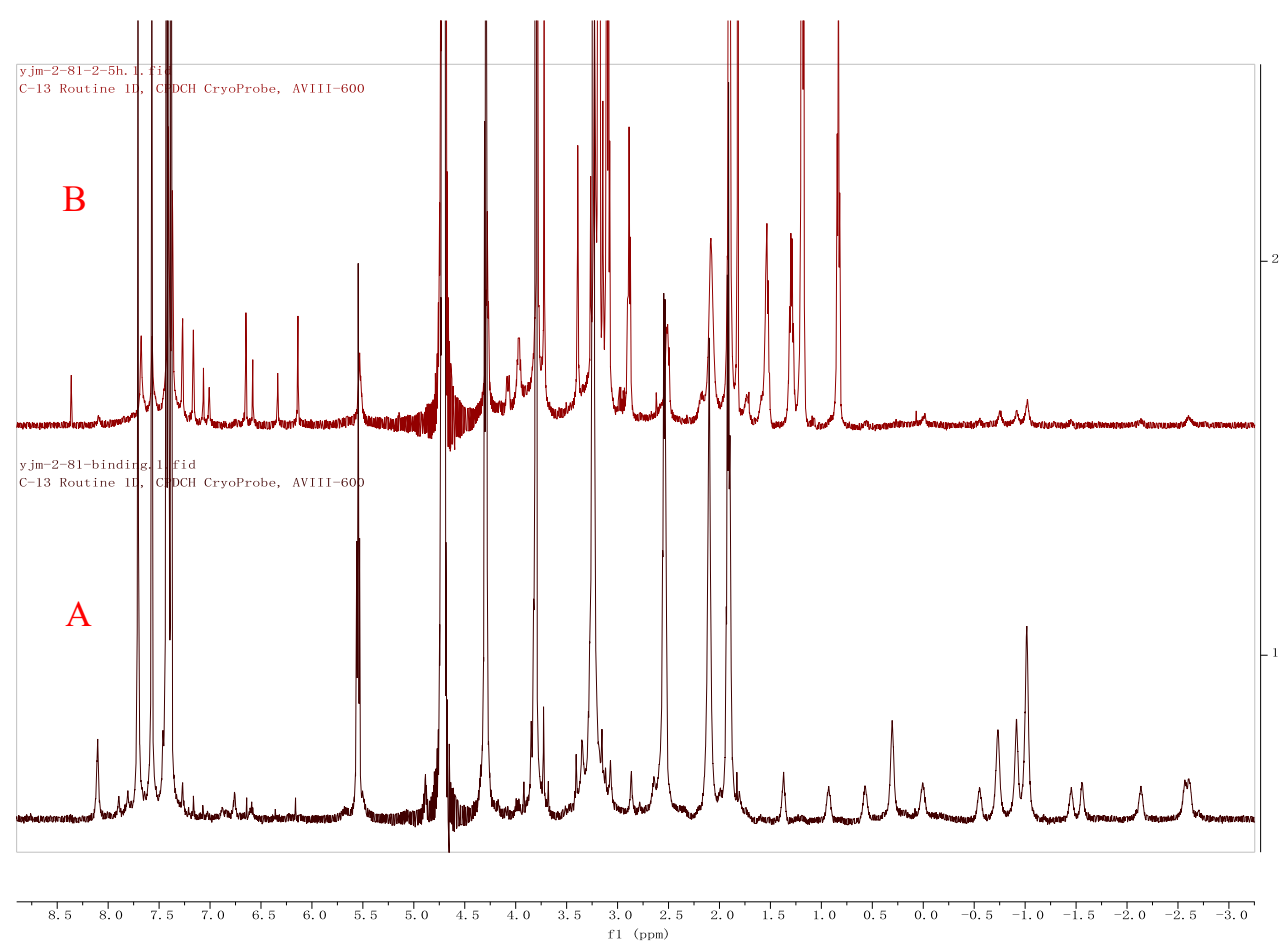

Figure S4 ${ }^{1} \mathrm{H}$ NMR spectra $\left(600 \mathrm{MHz}, \mathrm{D}_{2} \mathrm{O}, 298 \mathrm{~K}\right)$ : (A) 2a@cavitand 1(2.0 mM); (B) reacted for $15 \mathrm{~h}$.

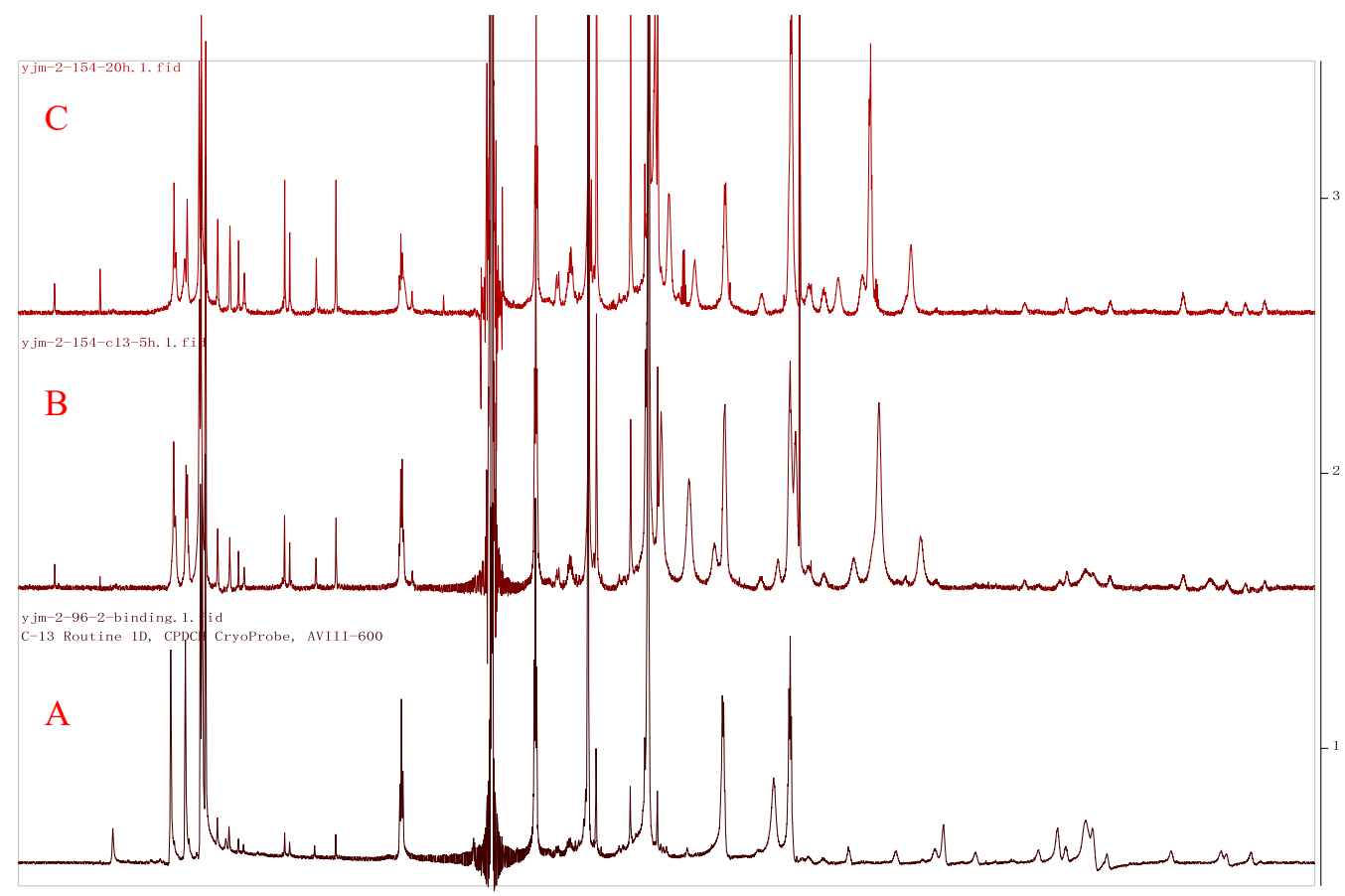

Figure S5 ${ }^{1} \mathrm{H}$ NMR spectra (600 MHz, D $\left.2 \mathrm{O}, 298 \mathrm{~K}\right)$ : (A) 2b@cavitand 1(2.0 mM); (B) reacted for $5 \mathrm{~h} ;(\mathbf{C})$ reacted for $20 \mathrm{~h}$. 


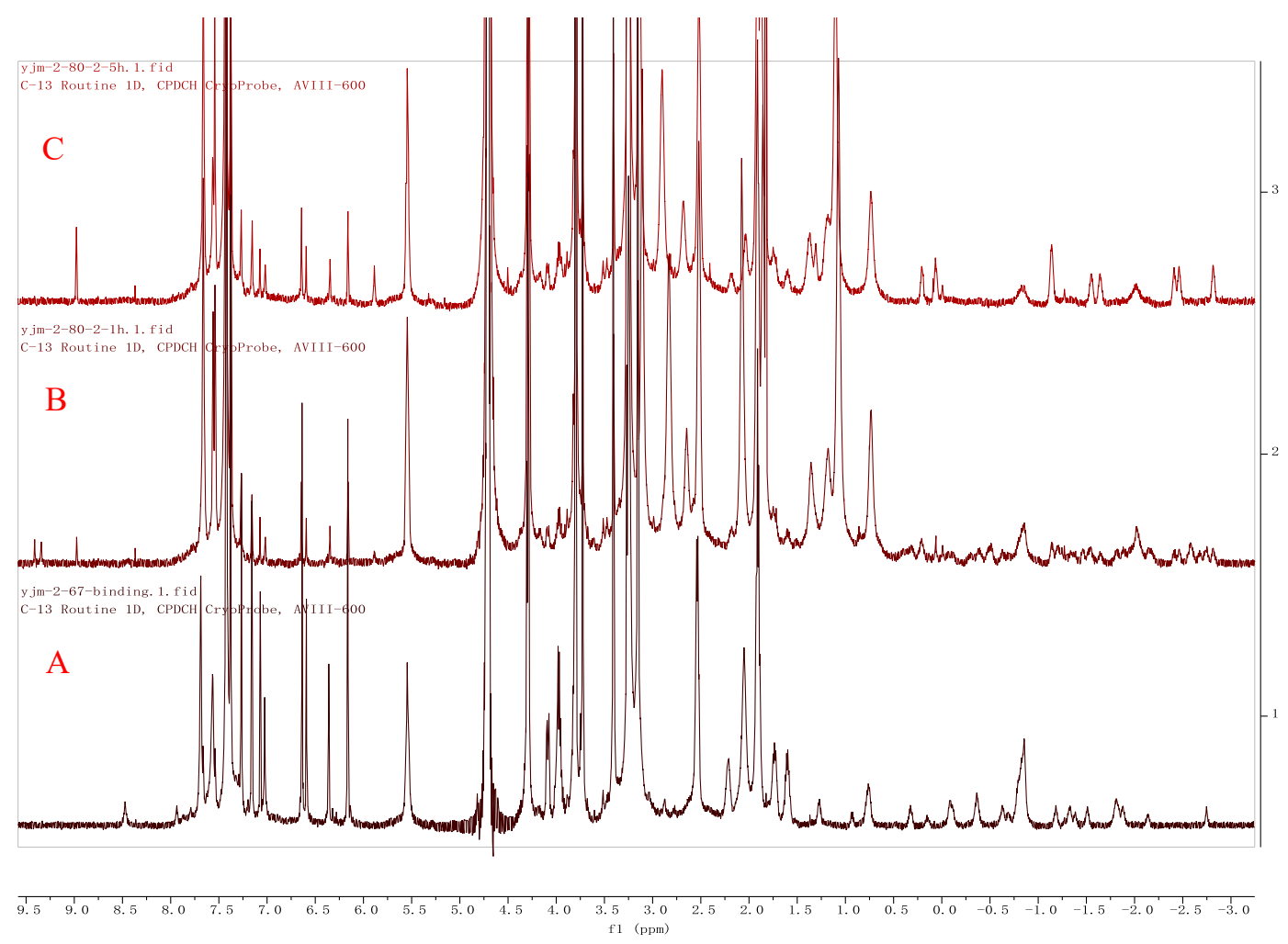

Figure S6 ${ }^{1} \mathrm{H}$ NMR spectra (600 MHz, D $\left.2 \mathrm{O}, 298 \mathrm{~K}\right)$ : (A) 2c@cavitand 1 (2.0 mM); (B) reacted for $1 \mathrm{~h}$; $(\mathbf{C})$ reacted for $5 \mathrm{~h}$.

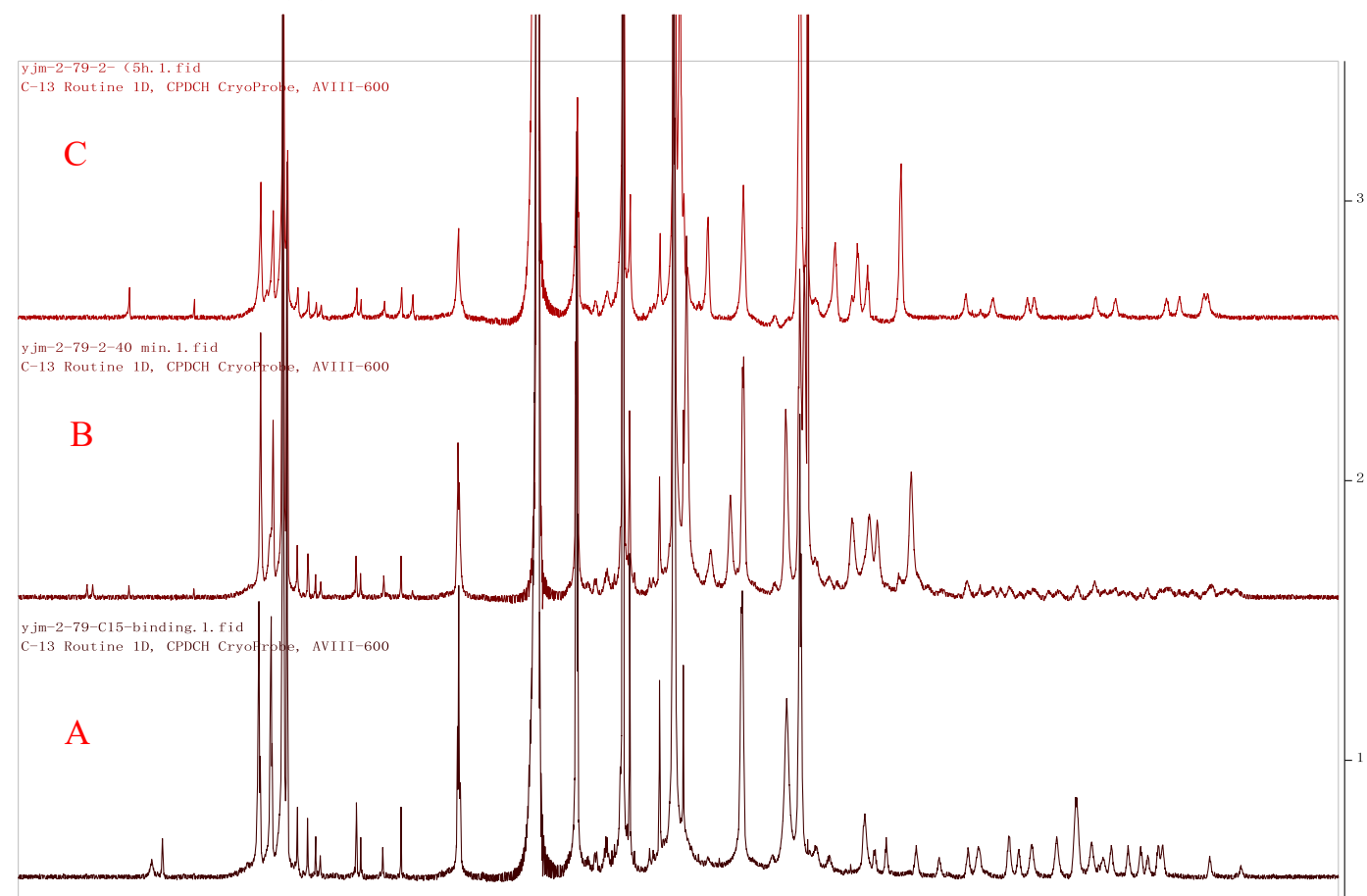

\begin{tabular}{lllllllllllllllllll}
10.0 & 9.5 & 9.0 & 8.5 & 8.0 & 7.5 & 7.0 & 6.5 & 6.0 & 5.5 & 5.0 & 4.5 & 4.0 & 3.5 & 3.0 \\
\hline
\end{tabular}

Figure S7 ${ }^{1} \mathrm{H}$ NMR spectra (600 MHz, D $\left.2 \mathrm{O}, 298 \mathrm{~K}\right)$ : (A) 2d@cavitand 1 (2.0 mM); (B) reacted for $40 \mathrm{~min}$; (C) reacted for $5 \mathrm{~h}$. 

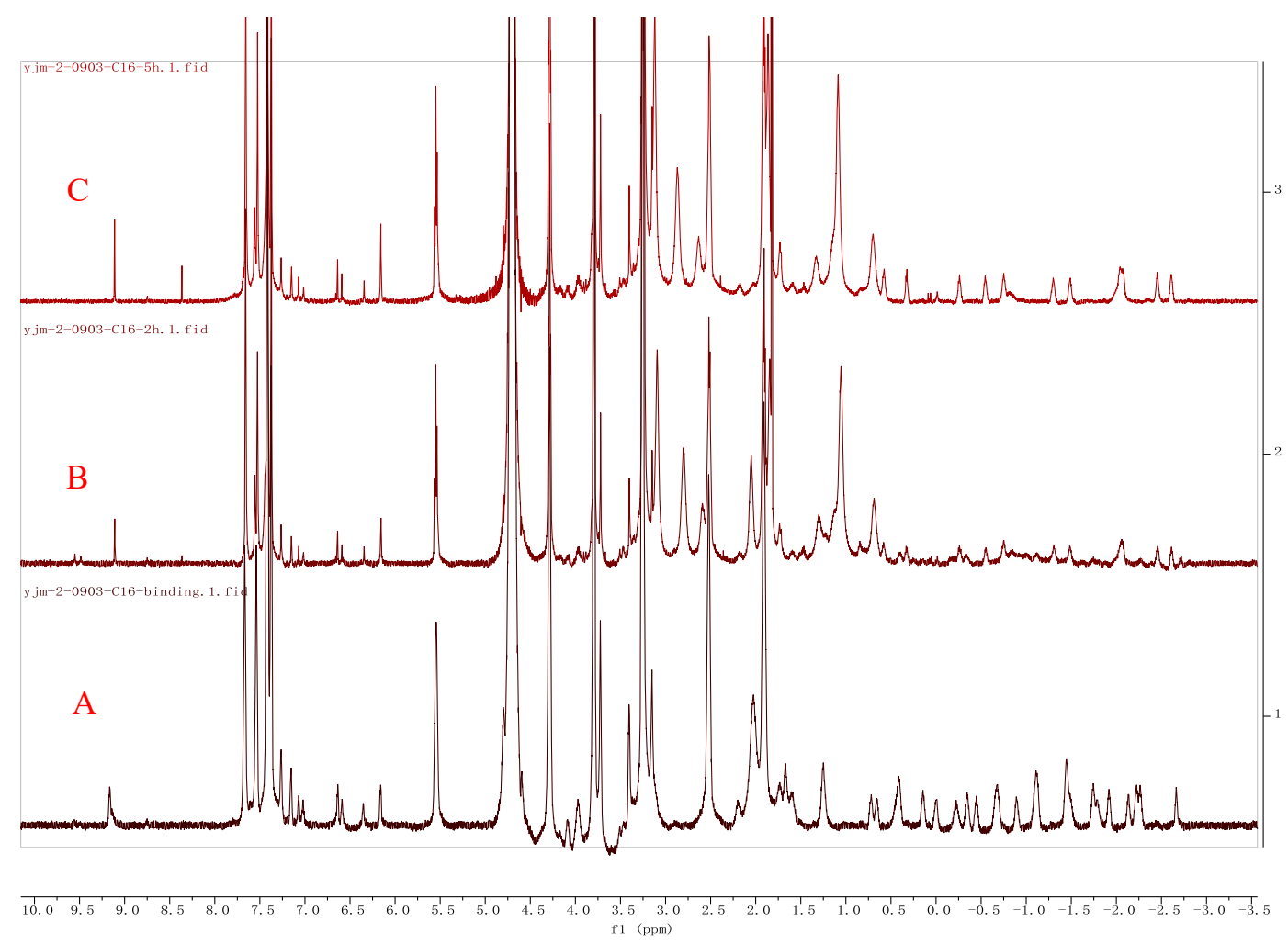

Figure S8 ${ }^{1} \mathrm{H}$ NMR spectra (600 MHz, D $\left.2 \mathrm{O}, 298 \mathrm{~K}\right)$ : (A) 2e@cavitand 1 (2.0 mM); (B) reacted for $2 \mathrm{~h}$; (C) reacted for $5 \mathrm{~h}$.

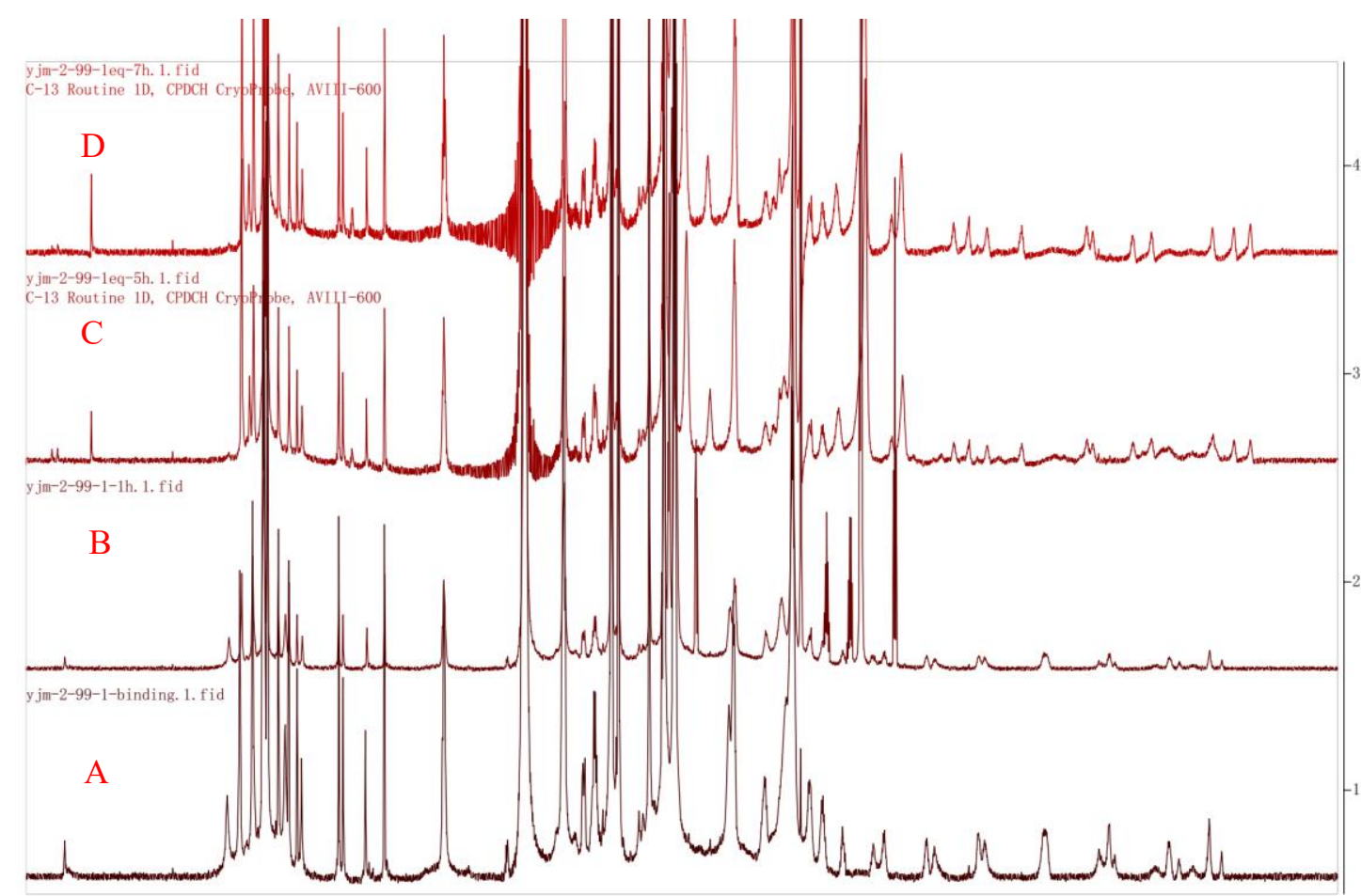

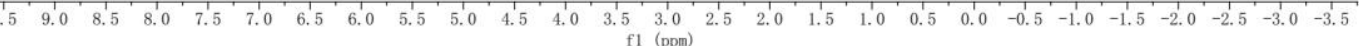

Figure S9 ${ }^{1} \mathrm{H}$ NMR spectra (600 MHz, D $\left.2 \mathrm{O}, 298 \mathrm{~K}\right)$ : (A) 2f@cavitand 1 (2.0 mM); (B) reacted for $1 \mathrm{~h}$; (C) reacted for $5 \mathrm{~h}$; (D) reacted for $7 \mathrm{~h}$. 


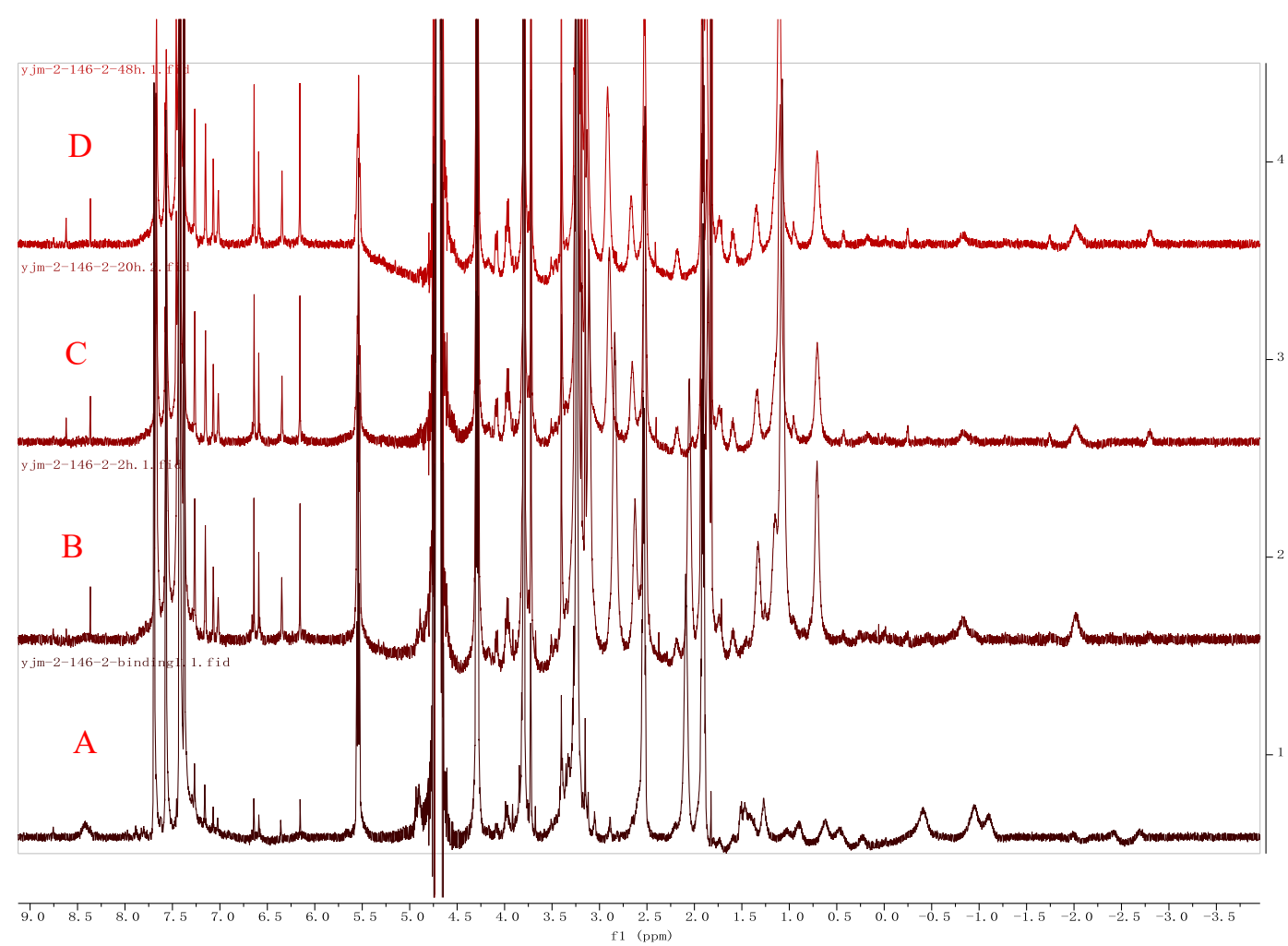

Figure S10 ${ }^{1} \mathrm{H}$ NMR spectra (600 MHz, D $\left.2 \mathrm{O}, 298 \mathrm{~K}\right)$ : (A) 2g@cavitand 1 (2.0 mM); (B) reacted for $2 \mathrm{~h}$; (C) reacted for $20 \mathrm{~h}$; (D) reacted for $48 \mathrm{~h}$.

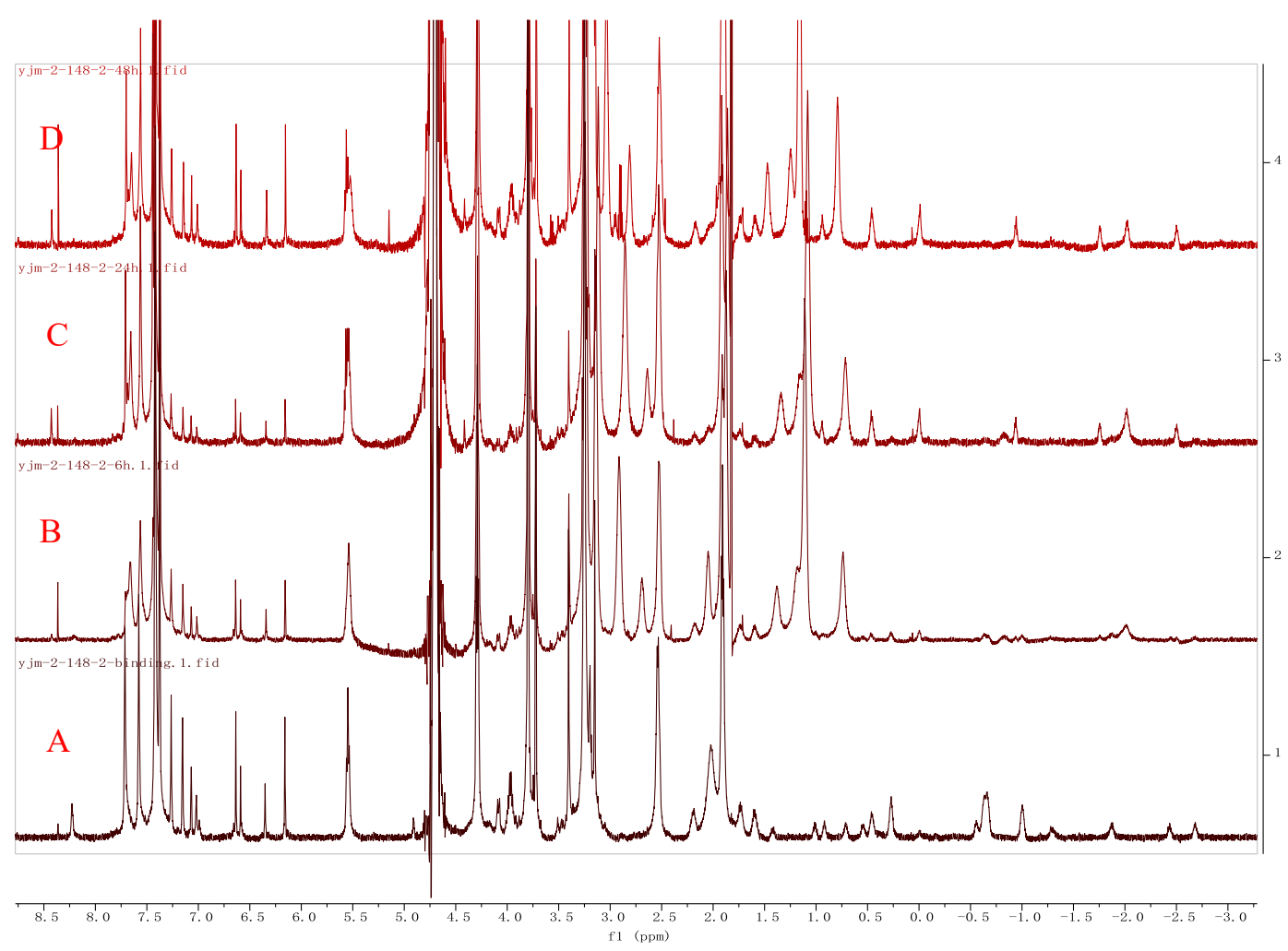

Figure S11 ${ }^{1} \mathrm{H}$ NMR spectra (600 MHz, D $\left.2 \mathrm{O}, 298 \mathrm{~K}\right)$ : (A) 2h@cavitand 1 (2.0 mM); (B) reacted for $6 \mathrm{~h}$; (C) reacted for $24 \mathrm{~h}$; (D) reacted for $48 \mathrm{~h}$. 


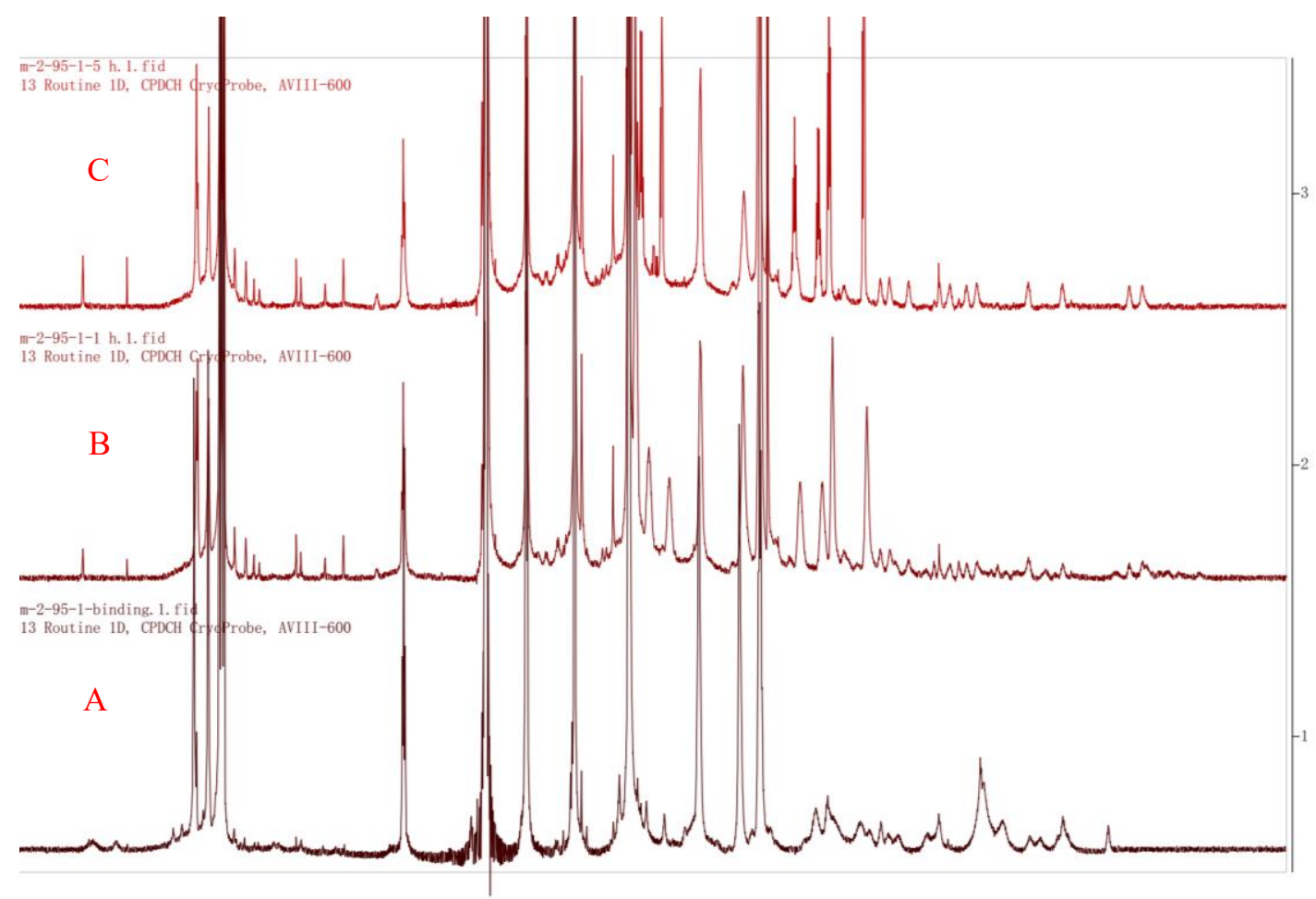

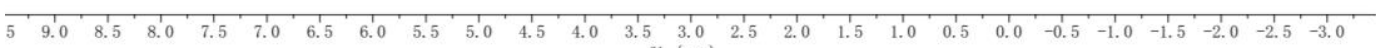

Figure S12 ${ }^{1} \mathrm{H}$ NMR spectra (600 MHz, D2O, $\left.298 \mathrm{~K}\right)$ : (A) 2i@cavitand 1 (2.0 mM); (B) reacted for $1 \mathrm{~h}$; (C) reacted for $5 \mathrm{~h}$.

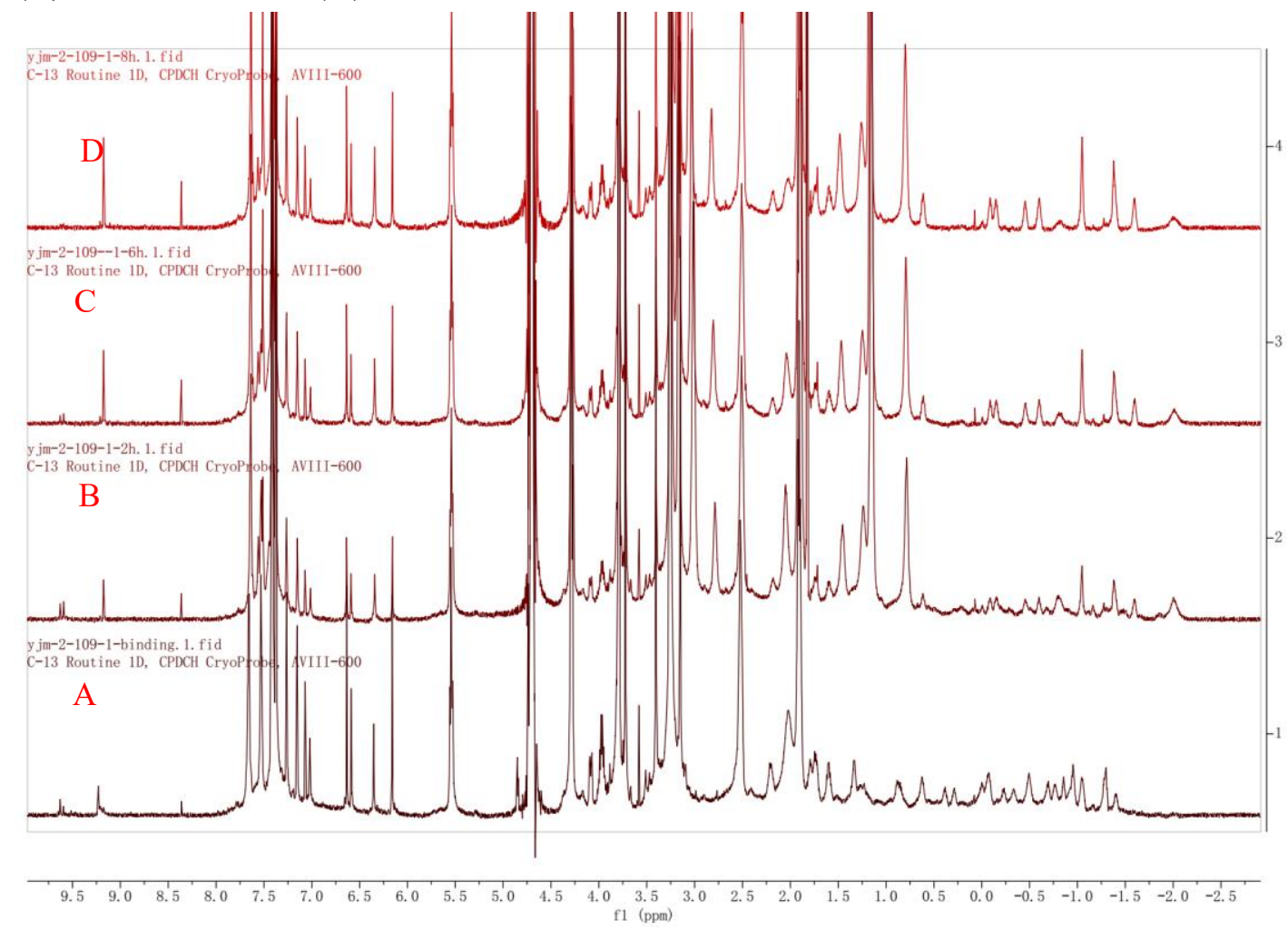

Figure S13 ${ }^{1} \mathrm{H}$ NMR spectra (600 MHz, $\left.\mathrm{D}_{2} \mathrm{O}, 298 \mathrm{~K}\right)$ : (A) 2j@cavitand 1 (2.0 mM); (B) reacted for $2 \mathrm{~h}$; (C) reacted for $6 \mathrm{~h}$; (D) reacted for $8 \mathrm{~h}$. 


\section{Recovery of Cavitand Study.}

After the cyclization reaction of $\mathbf{2 e}$ was finished, the product was removed by extraction with $\mathrm{CDCl}_{3}$. Then the aqueous phase was concentrated in vacuo and the residue was suspended in acetone and heated under reflux for $6 \mathrm{~h}$. The recovered cavitand was collected by filtration and washed with acetone. After drying under high vacuum, the recovered cavitand $\mathbf{1}$ was obtained as a pale powder.
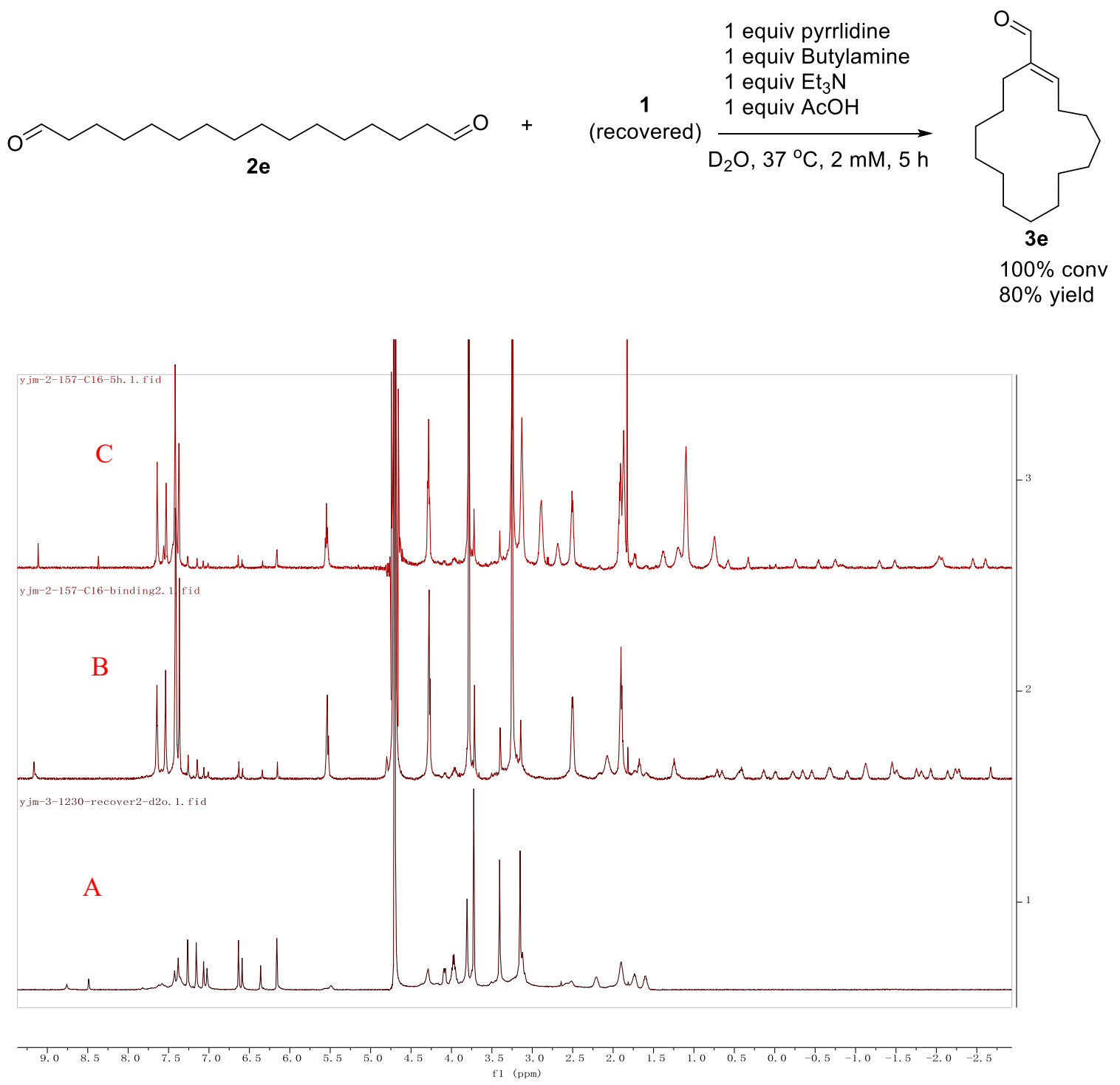

Figure S14 ${ }^{1} \mathrm{H}$ NMR spectra $\left(600 \mathrm{MHz}, \mathrm{D}_{2} \mathrm{O}, 298 \mathrm{~K}\right)$ : (A) recovered cavitand 1; (B) $\mathbf{2 e} @$ recovered cavitand $\mathbf{1}(2.0 \mathrm{mM})$; (C) reacted for $5 \mathrm{~h}$. 


\section{Determination of the Binding Constant $\left(K_{\mathrm{A}}\right)$}

Experimental Procedure: Titration of $3 \mathbf{c}(1 \mathrm{mM})$ in $0.5 \mathrm{~mL}$ of $\mathrm{D}_{2} \mathrm{O}$ was performed adding different volumes $(3,4,5,6,7$ and $8 \mu \mathrm{L})$ of $50 \mathrm{mM}$ guest's solution in DMSOd6, corresponding to $30,40,50,60,70$ and $80 \%$ of the host, respectively. Dimethylsulfone ( $5 \mu \mathrm{L}$ from $100 \mathrm{mM} \mathrm{D}_{2} \mathrm{O}$ solution, ratio $1: 1$ with host $1, \delta=3.15 \mathrm{ppm}$ ) was used as internal standard for the calculation of the involved species' concentration (See example below). ${ }^{1} \mathrm{H}$ NMR spectra are recorded after $1 \mathrm{~h}$ of sonication at room temperature. The reported binding constant $\left(\mathrm{K}_{\mathrm{A}}\right)$ value results to be the average of two indipendent titration. The releated error for each calculated $\mathrm{K}_{\mathrm{A}}$ value is maximum of $15 \%$.

$$
\begin{gathered}
\mathbf{H}+\mathbf{G} \stackrel{\mathrm{K}_{\mathrm{A}}}{\rightleftharpoons} \mathbf{H G} \\
\text { Binding Constant }=\frac{[\mathrm{HG}]}{[\mathrm{H}][\mathrm{G}]}
\end{gathered}
$$

Figure S15: Equilibrium between a general host $(\mathrm{H})$ and a general guest $(\mathrm{G})$ and the formed host-guest complex (HG) and relative mathematical formula for the binding constant $\left(\mathrm{K}_{\mathrm{A}}\right)$ calculation.

Example for Titration of 3c (See S16):

\begin{tabular}{|l|l|l|l|}
\hline Entry & Guest's equivalent (eq) & Guest Peak's Area & $\mathbf{K}_{\mathbf{A}}\left(\mathbf{M}^{-1}\right)$ \\
\hline 1 & 0.3 & 0.027 & 402 \\
\hline 2 & 0.4 & 0.036 & 414 \\
\hline 3 & 0.5 & 0.044 & 413 \\
\hline 4 & 0.6 & 0.053 & 428 \\
\hline 5 & 0.7 & 0.060 & 422 \\
\hline 6 & 0.8 & 0.069 & 440 \\
\hline
\end{tabular}

$\mathrm{K}_{\mathrm{A}}$ Calculation for 0.3 eq of $3 \mathrm{c}$ (Entry 1, Table above) $\mathbf{C}_{0}$ Guest: $0.3 \mathrm{mmol}$

Co Host: 1 mmol

Co STD: 1 mmol

Bound Guest Peak Unit: Peak Area (See Table) / $\mathrm{N}^{\circ}$ hydrogens: 0.027/2 $=0.0135$

Standard Peak Unit: Peak Area (Arbitrary) / $\mathrm{N}^{\circ}$ hydrogens: $1 / 6=0.1666$

Ratio STD/ Bound Guest: STD Unit / Bound Guest Unit: 0.166 / $0.027=12.35$

Conc. HG (mM): $\mathrm{C}_{0}$ STD / (Ratio STD/Bound Guest): $1 / 12.35=0.081$

Conc. G (mM): $\mathrm{C}_{0}$ Guest $-\mathrm{HG}=0.3-0.081=0.219$

Conc. $\mathbf{H}(\mathbf{m M}): \mathrm{C}_{0}$ Host $-\mathrm{HG}=1-0.081=0.919$

KA (mM-1): $0.402 \mathrm{mM}^{-1}$

KA (M-1): 402 M $^{-1}$ 


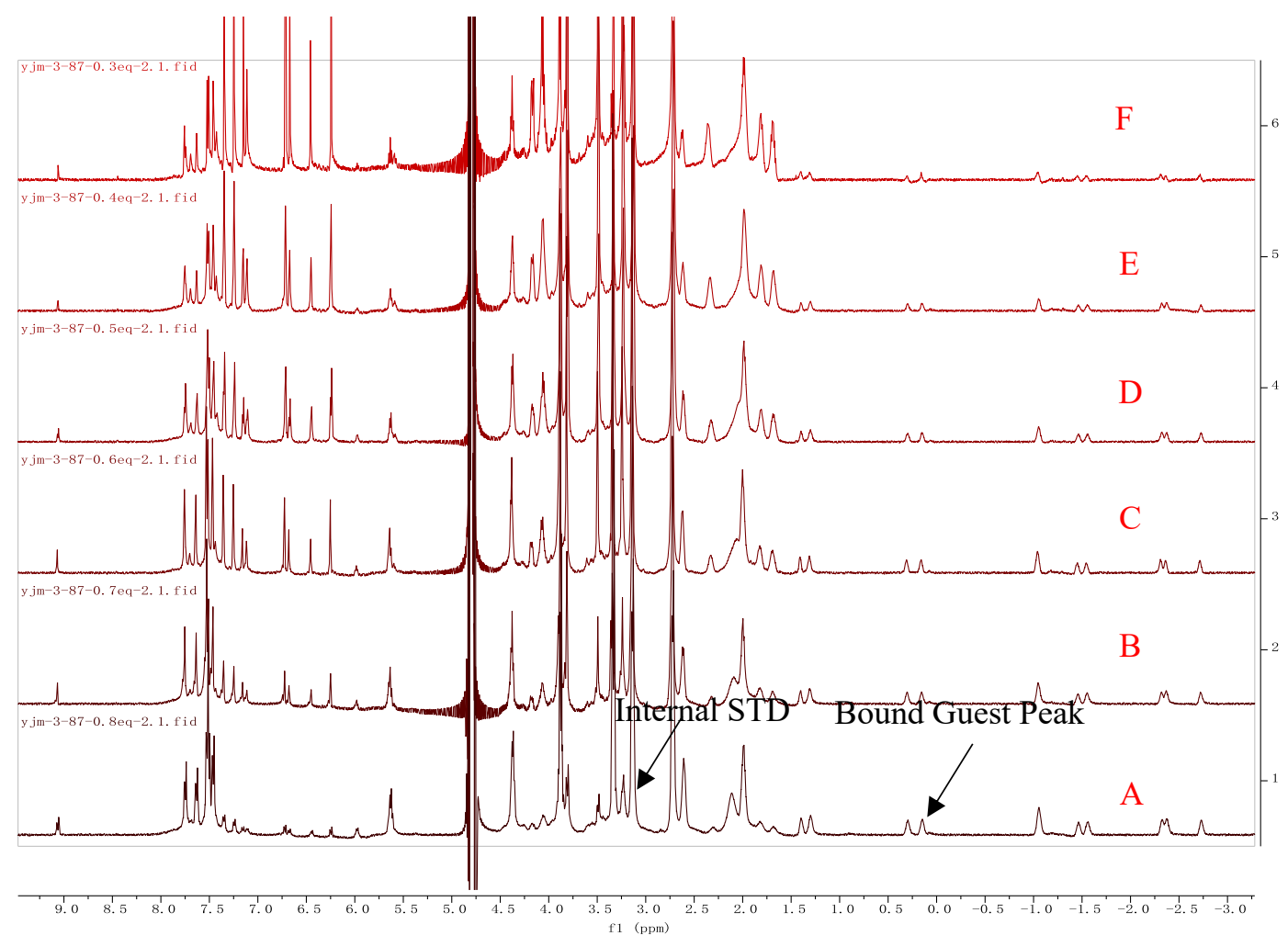

Figure S16: ${ }^{1} \mathrm{H}$ NMR spectra of 1 with different amount of 3c: (A) 0.3 eq. (B) 0.4 eq. (C) 0.5 eq. (D) 0.6 eq. (E) 0.7 eq. (F) and 0.8 eq. (G). Averaged $\mathrm{K}_{\mathrm{A}}$ : $420 \mathrm{M}^{-1}$.

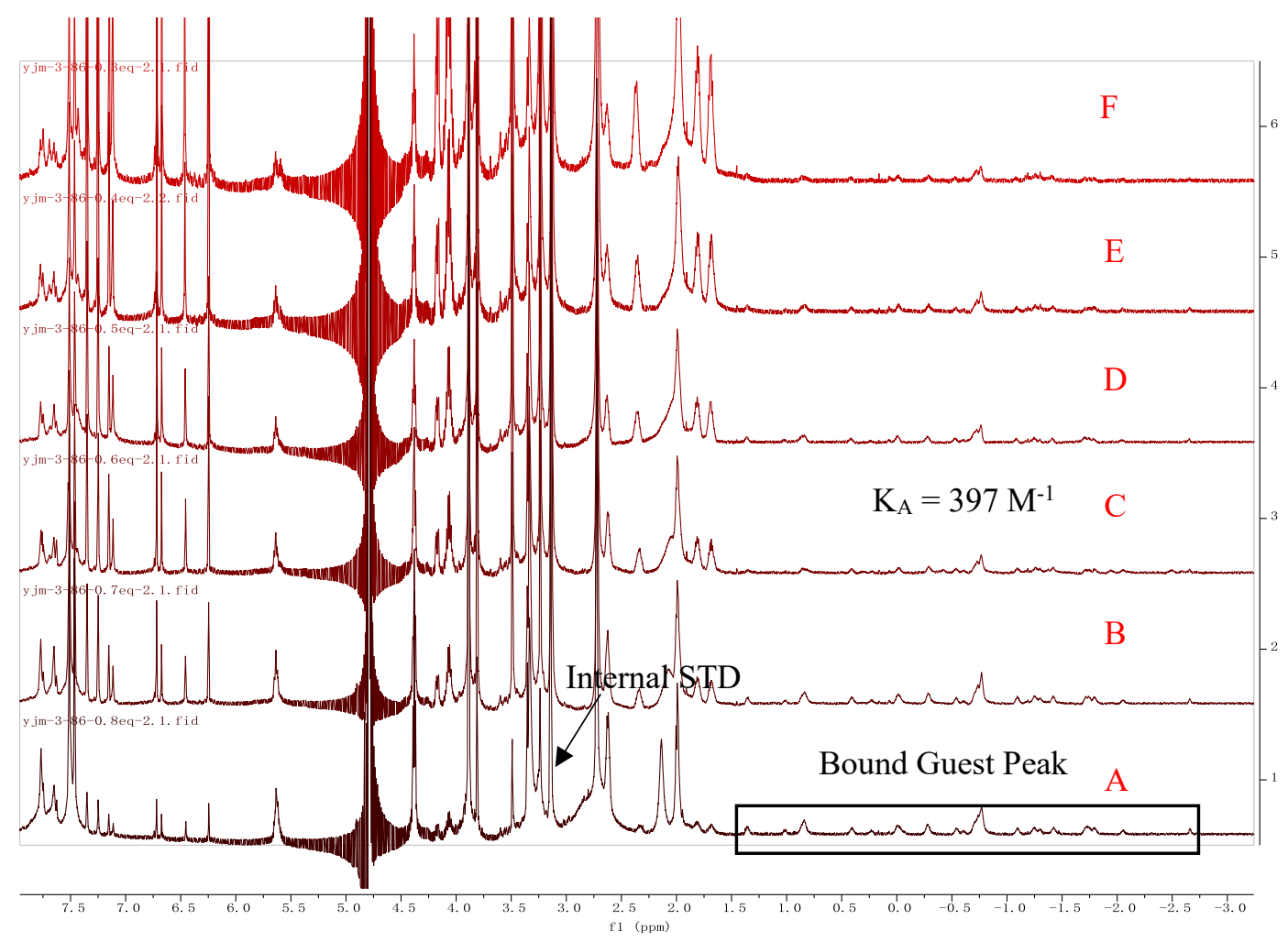

Figure S17: ${ }^{1} \mathrm{H}$ NMR spectra of 1 with different amount of 2c: (A) 0.3 eq. (B) 0.4 eq. (C) 0.5 eq. (D) 0.6 eq. (E) 0.7 eq. (F) and 0.8 eq. (G) Averaged $\mathrm{K}_{\mathrm{A}}: 397 \mathrm{M}^{-1}$. 


\section{Procedure for the Allyl Deuterated Product.}

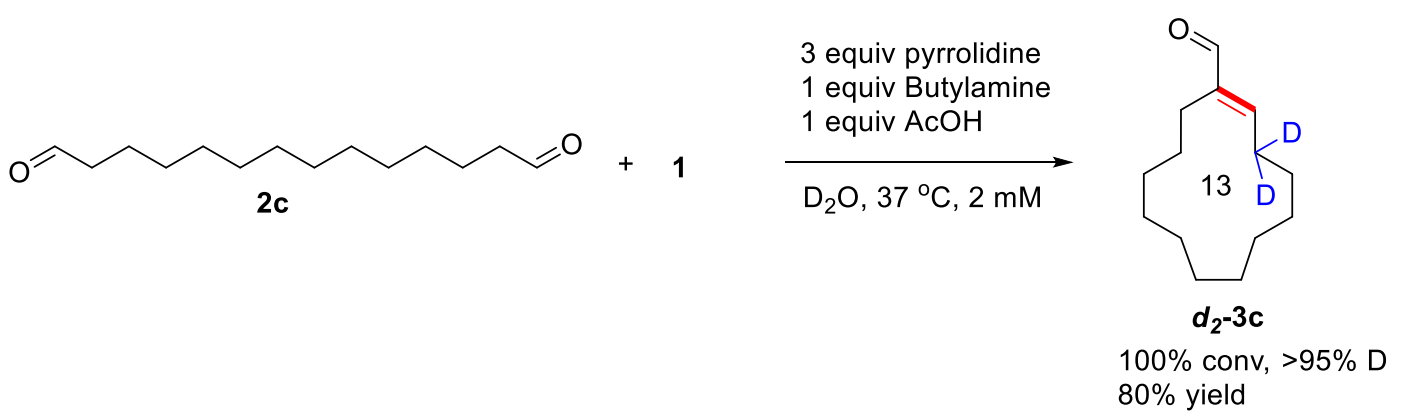

Under $\mathrm{N}_{2}$ atmosphere, tridecanedial $\mathbf{2 c}(8.5 \mathrm{mg}, 0.04 \mathrm{mmol})$ and $\mathbf{1}(67 \mathrm{mg}, 0.04 \mathrm{mmol})$ were introduced into a round bottom flask. Then $20 \mathrm{~mL} \mathrm{D}_{2} \mathrm{O}$ was injected into the flask in one portion. After stirring for $2 \mathrm{~h}$ at $37^{\circ} \mathrm{C}, \mathrm{Et}_{3} \mathrm{~N}$ ( $\left.4 \mathrm{mg}, 0.04 \mathrm{mmol}\right), \mathrm{AcOH}(2.4 \mathrm{mg}$, $0.04 \mathrm{mmol})$, pyrrolidine $(8.4 \mathrm{mg}, 0.12 \mathrm{mmol})$, and propylamine $(2.9 \mathrm{mg}, 0.04 \mathrm{mmol})$ were injected into the flask. The resulting mixture was stirred at $37^{\circ} \mathrm{C}$ for another $4 \mathrm{~h}$, then extracted with $\mathrm{CHCl}_{3}(20 \mathrm{~mL} \times 3)$. The combined organic phases were washed with brine and dried over $\mathrm{Na}_{2} \mathrm{SO}_{4}$. The filtrate was concentrated in vacuo and the residue was purified by flash column chromatography $(\mathrm{PE} / \mathrm{EA}=20: 1, \mathrm{v} / \mathrm{v})$.

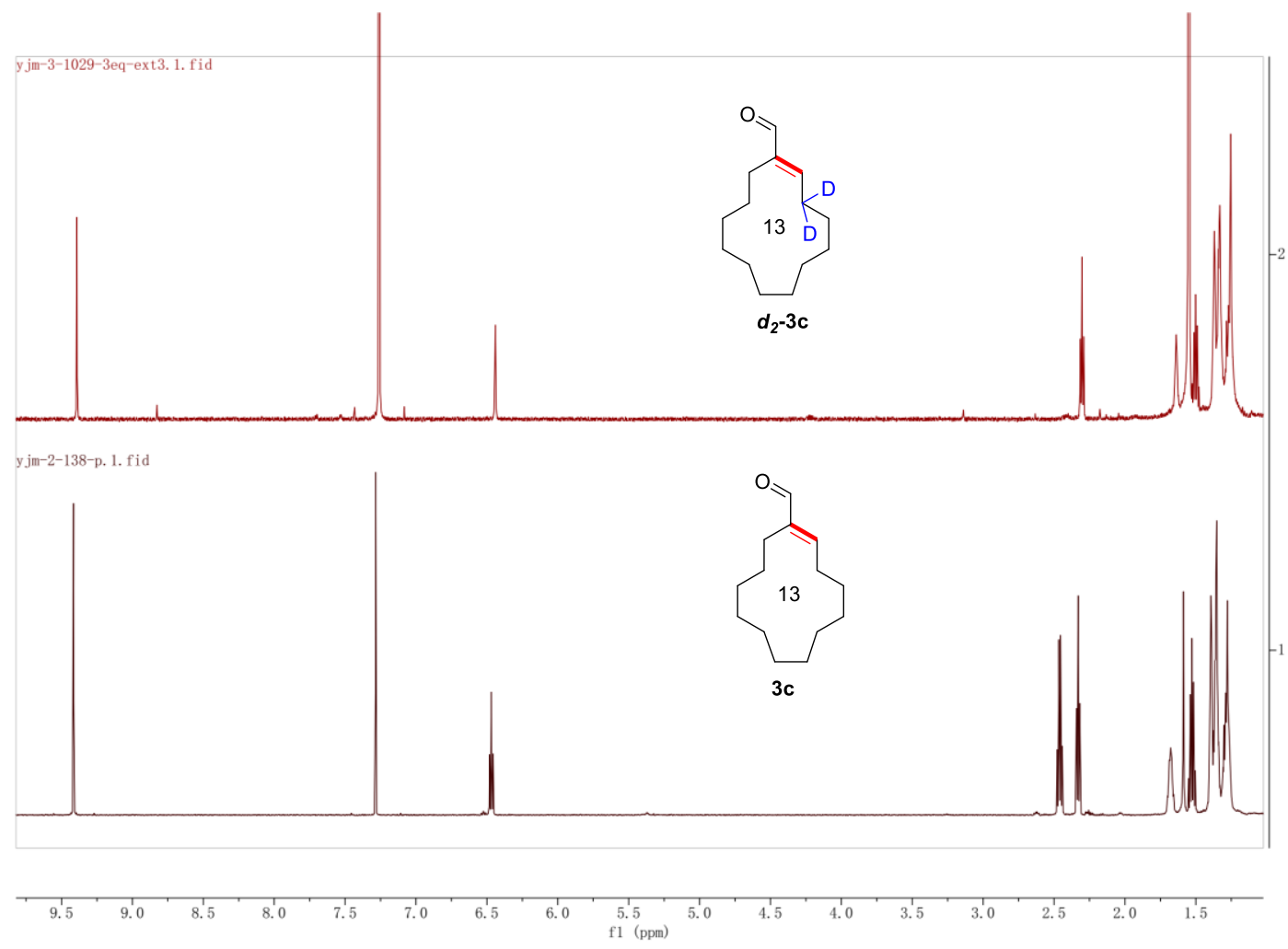

Figure S18: ${ }^{1} \mathrm{H}$ NMR spectra of $\boldsymbol{d}_{\mathbf{2}} \mathbf{- 3} \mathrm{c}$ and $\mathbf{3 c}$. 


\section{NMR Spectra of New Compounds.}

pentadecanedial 2d
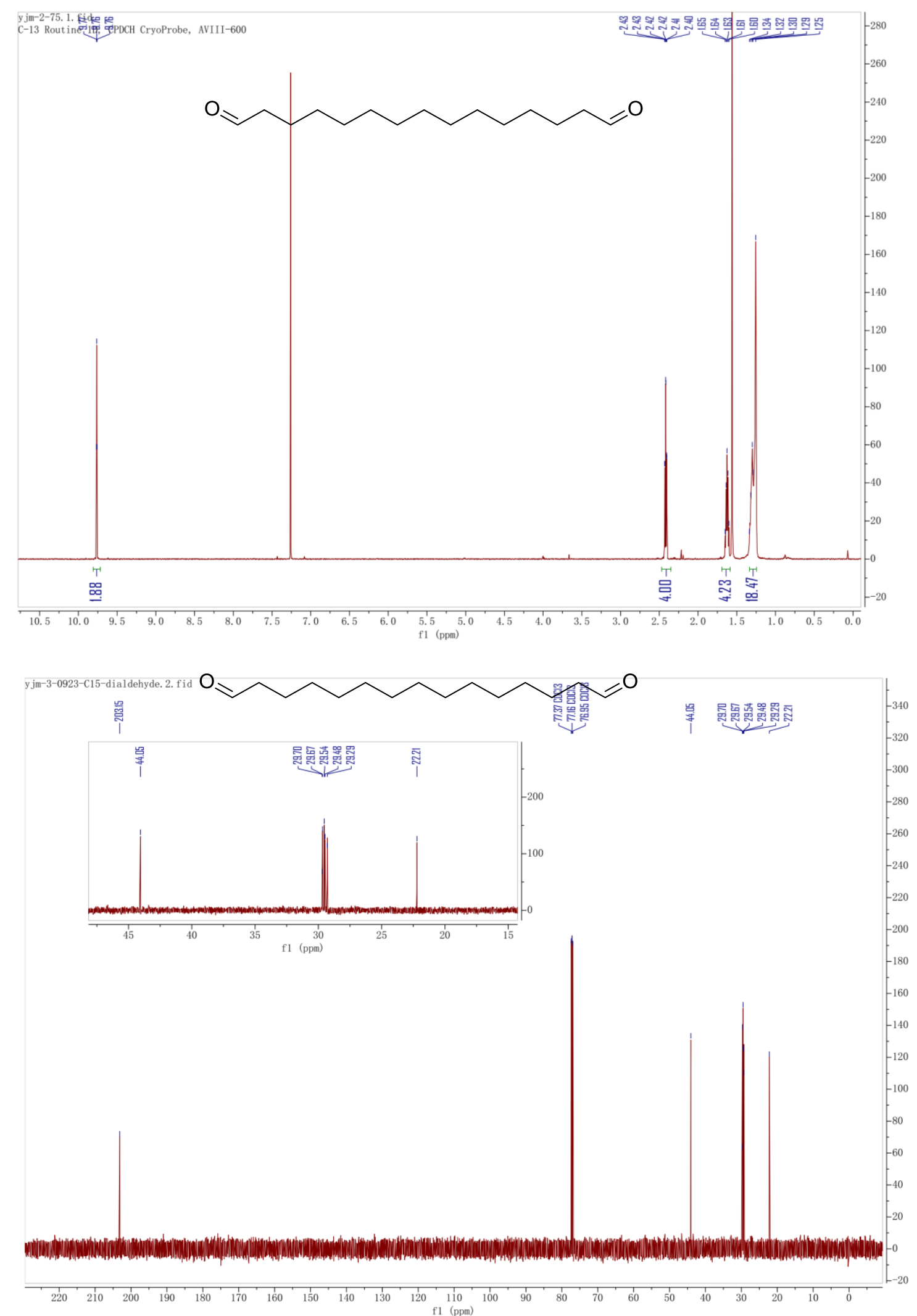
octadecanedial $\mathbf{2 f}$
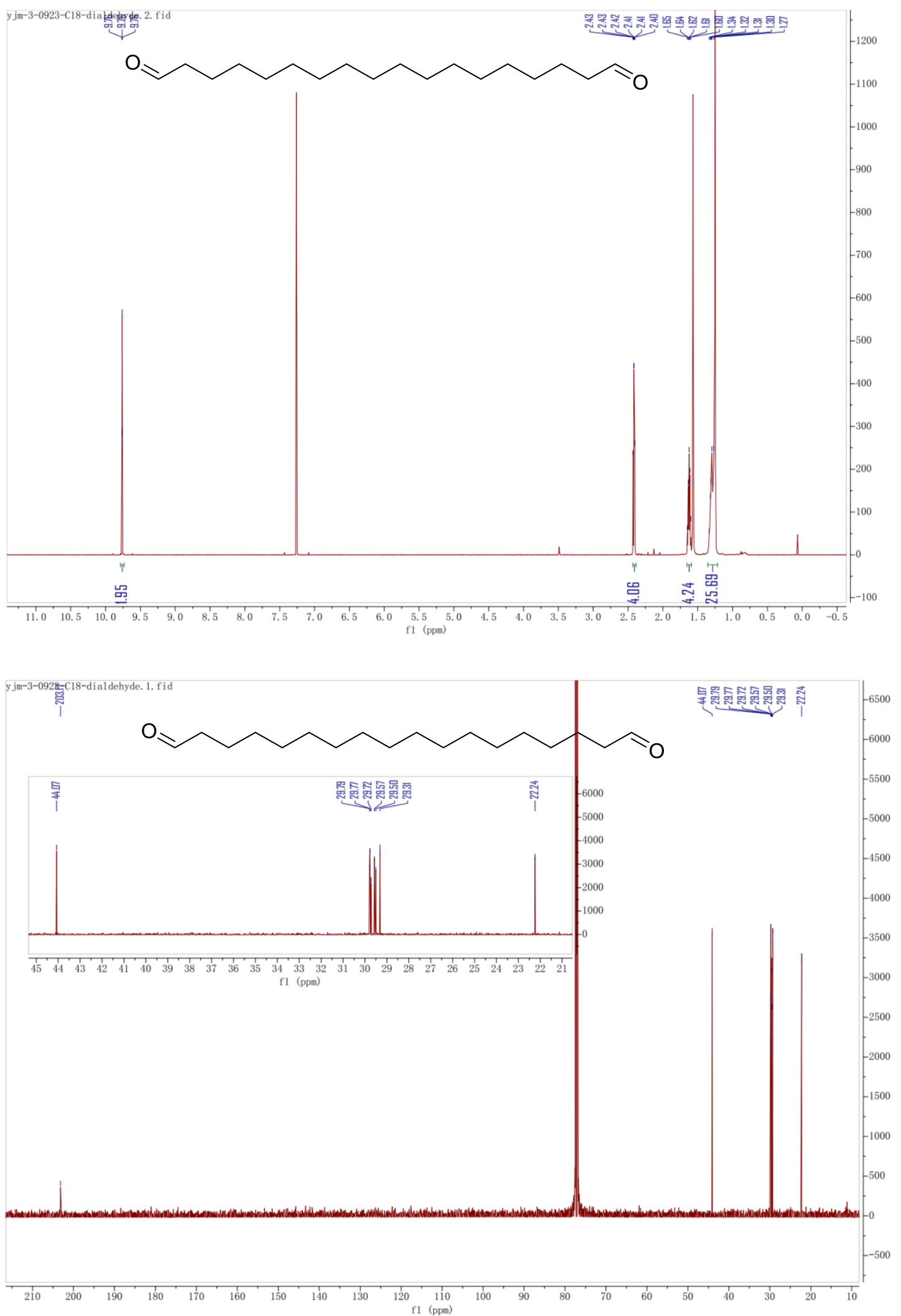
6,6'-oxydihexanal $\mathbf{2 g}$
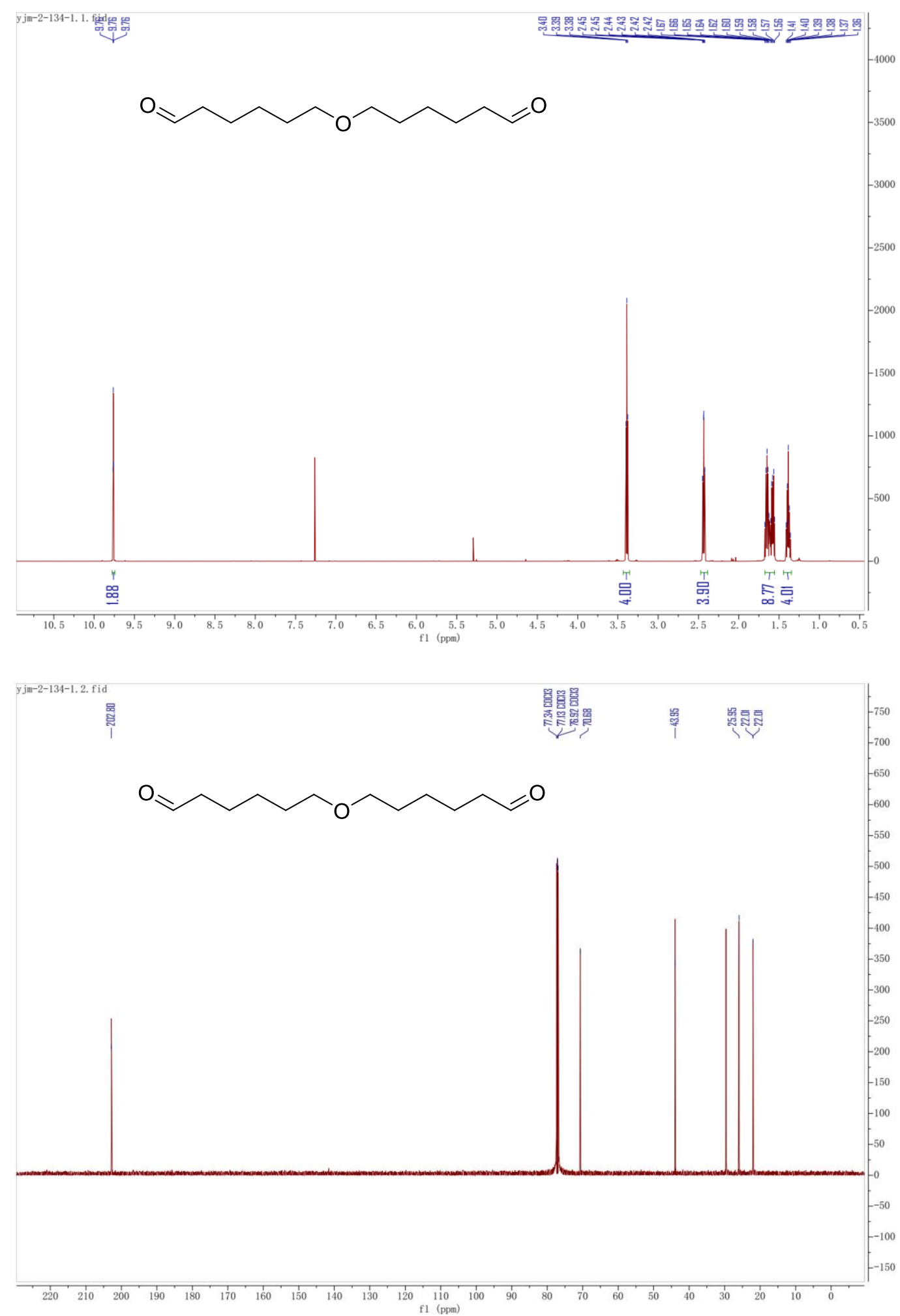
6,6'-thiodihexanal $\mathbf{2 h}$
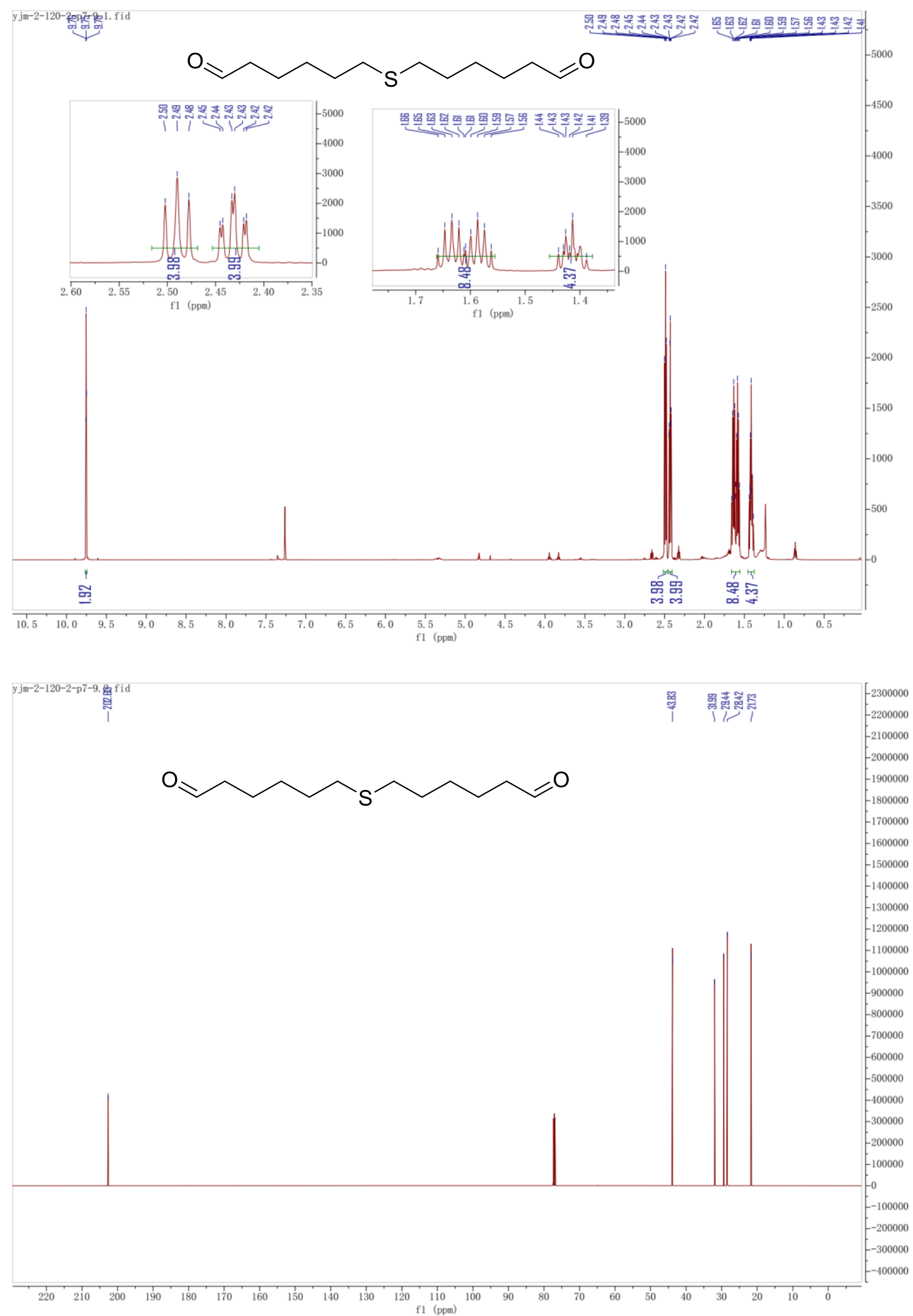
8,8'-oxydioctanal $\mathbf{2 i}$
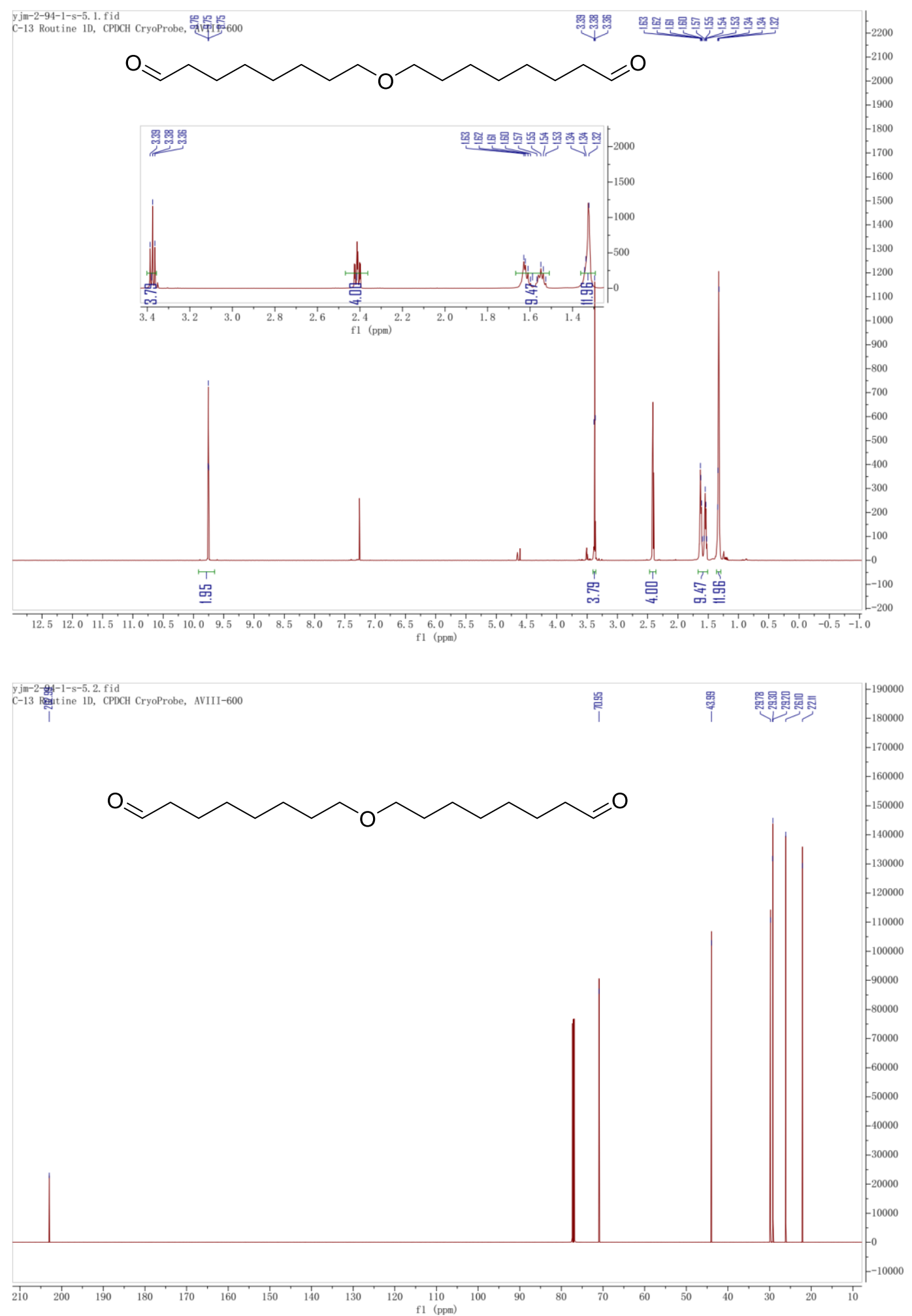


\section{8,8'-thiodioctanal $\mathbf{2 j}$}
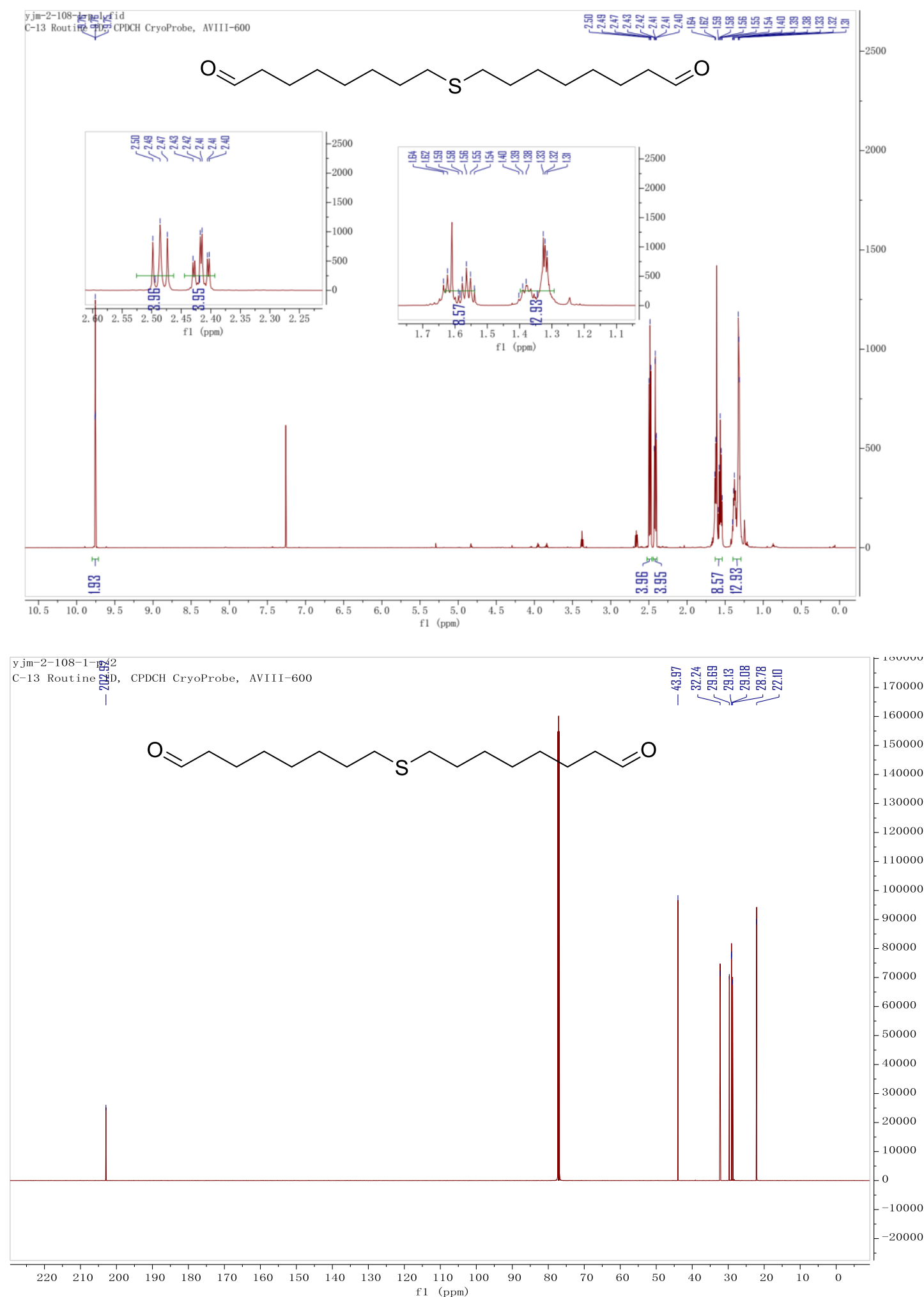
(1E,13E)-cyclotetracosa-1,13-diene-1,13-dicarbaldehyde 4
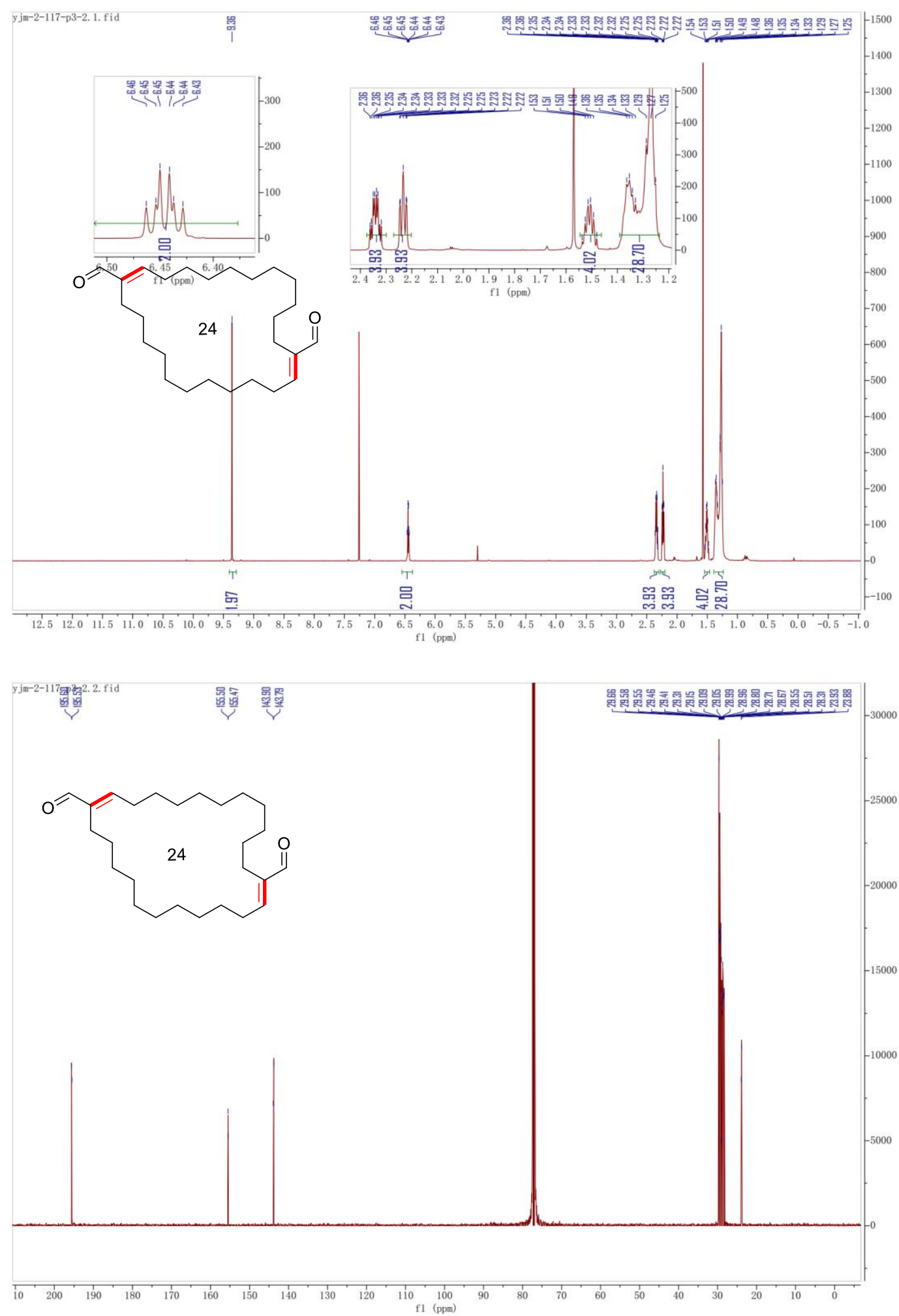
(E)-cyclododec-1-ene-1-carbaldehyde $\mathbf{3 b}$
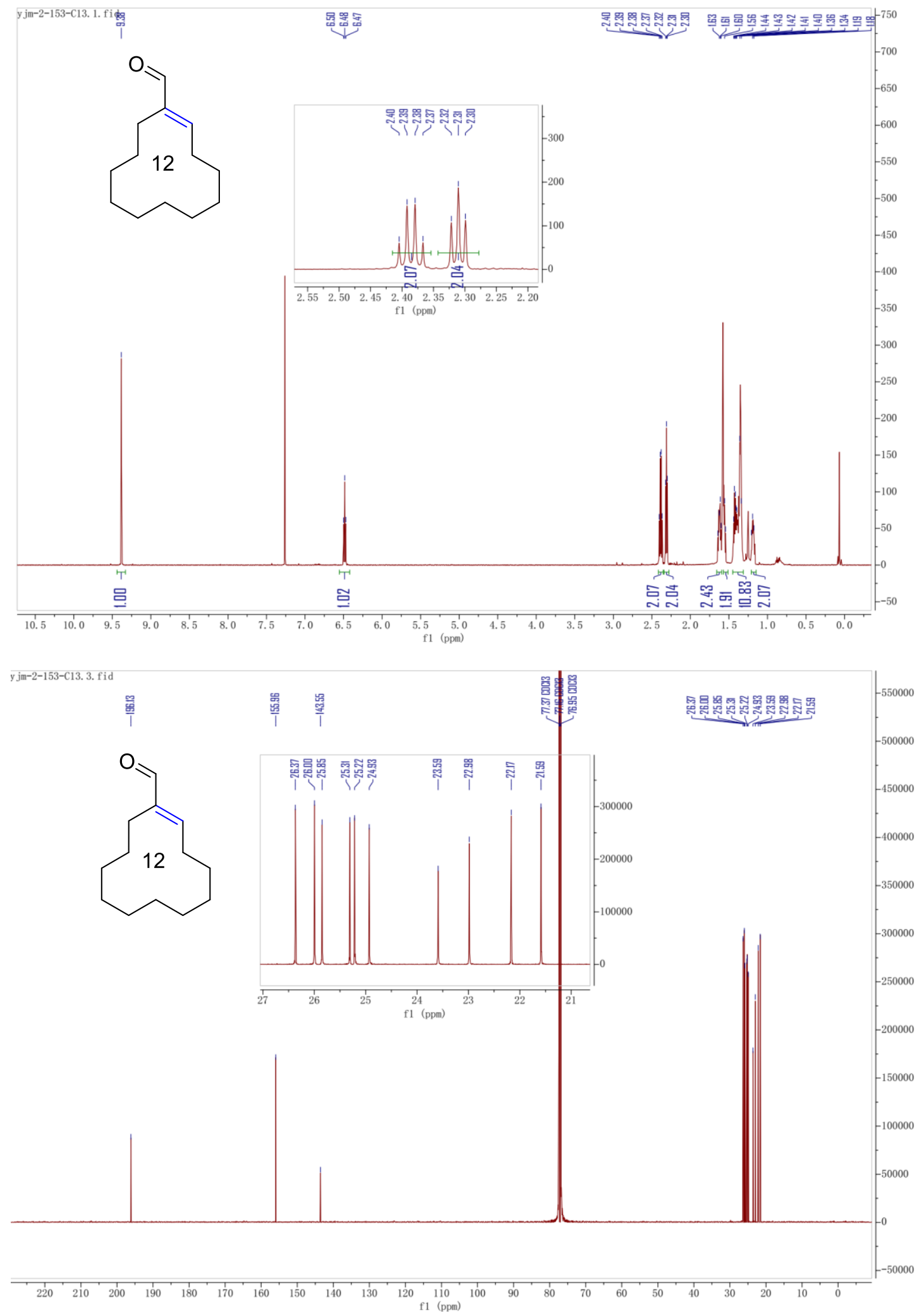
(E)-cyclotridec-1-ene-1-carbaldehyde 3c

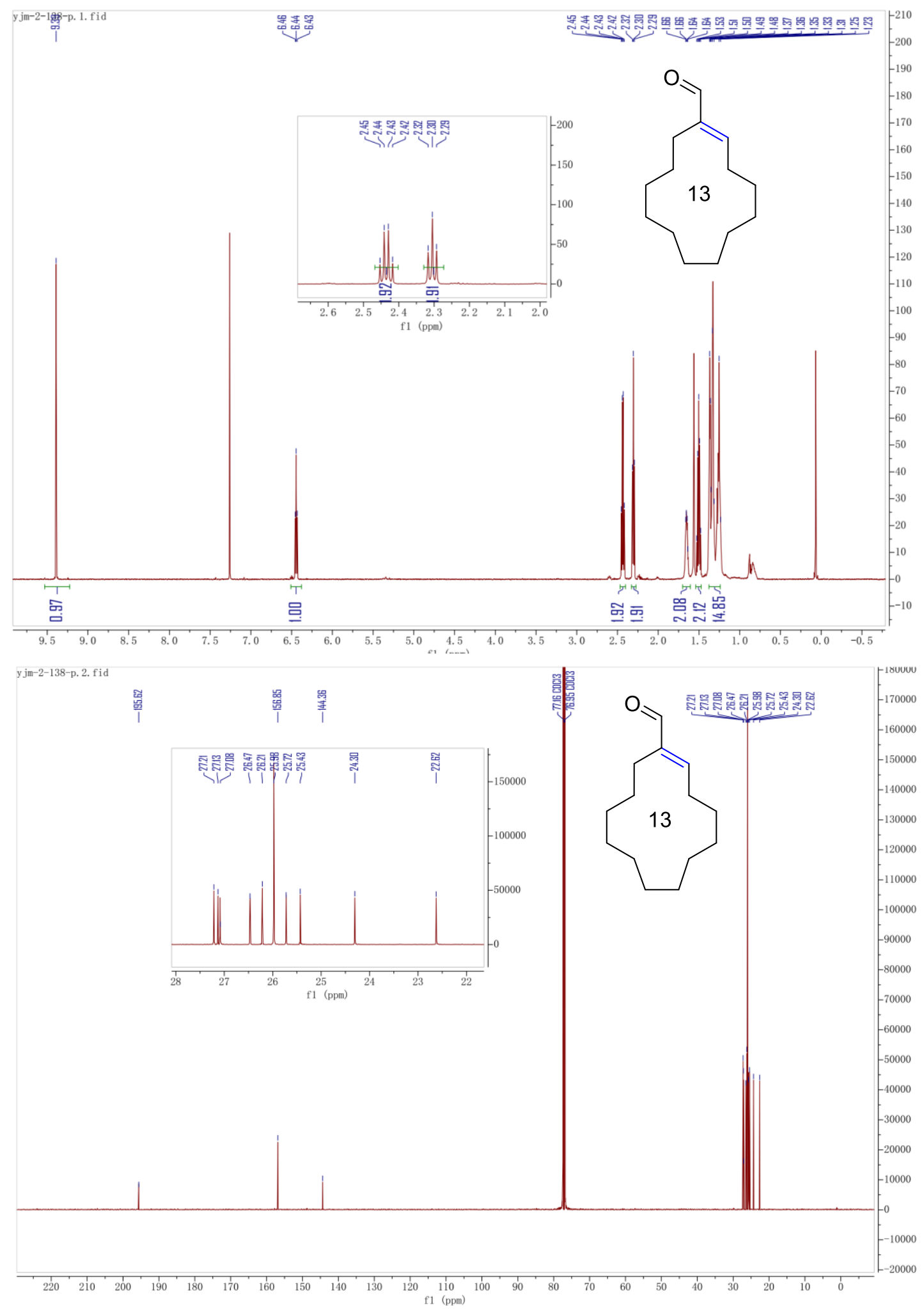


(E)-cyclotetradec-1-ene-1-carbaldehyde 3d
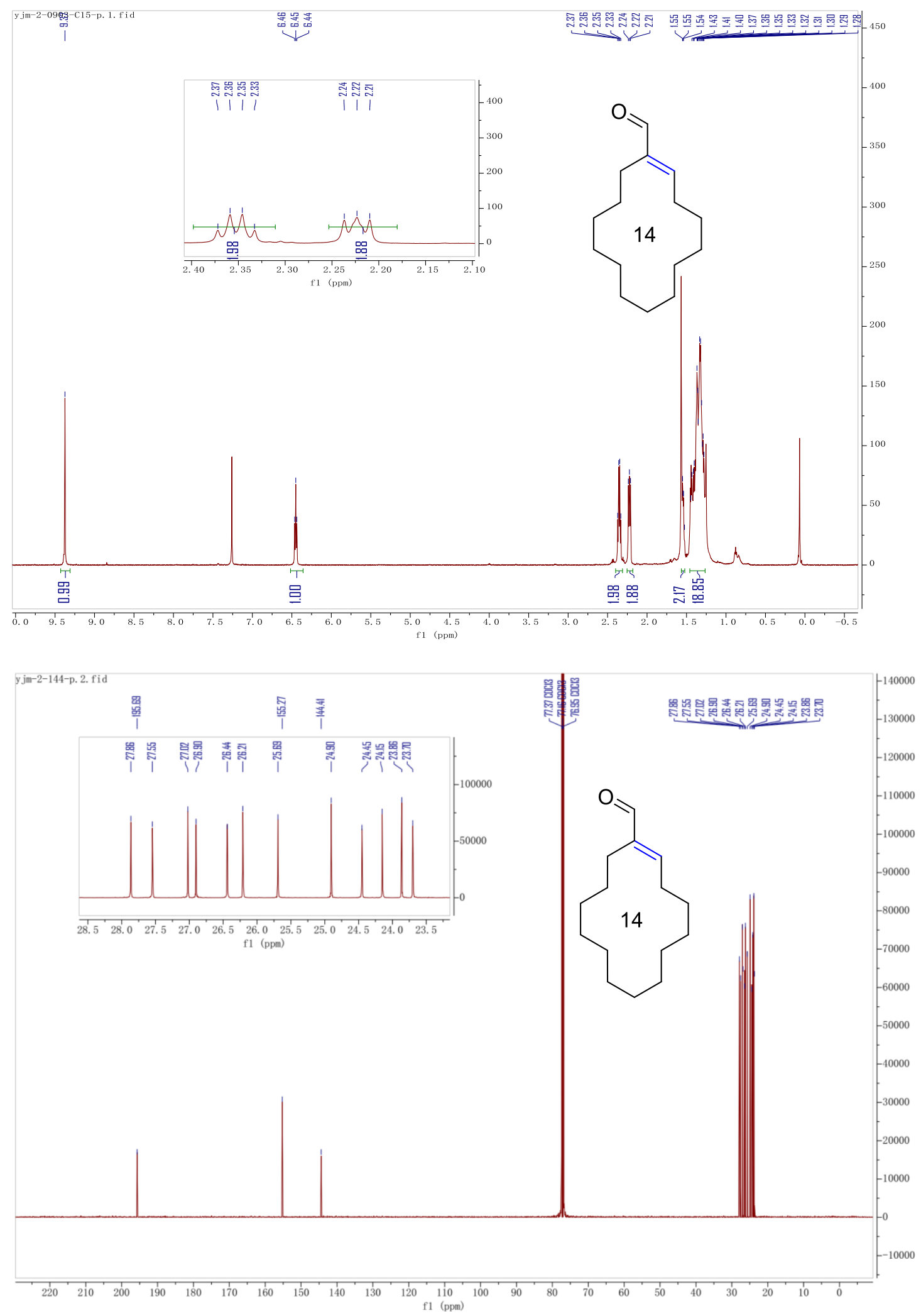
(E)-cyclopentadec-1-ene-1-carbaldehyde 3e
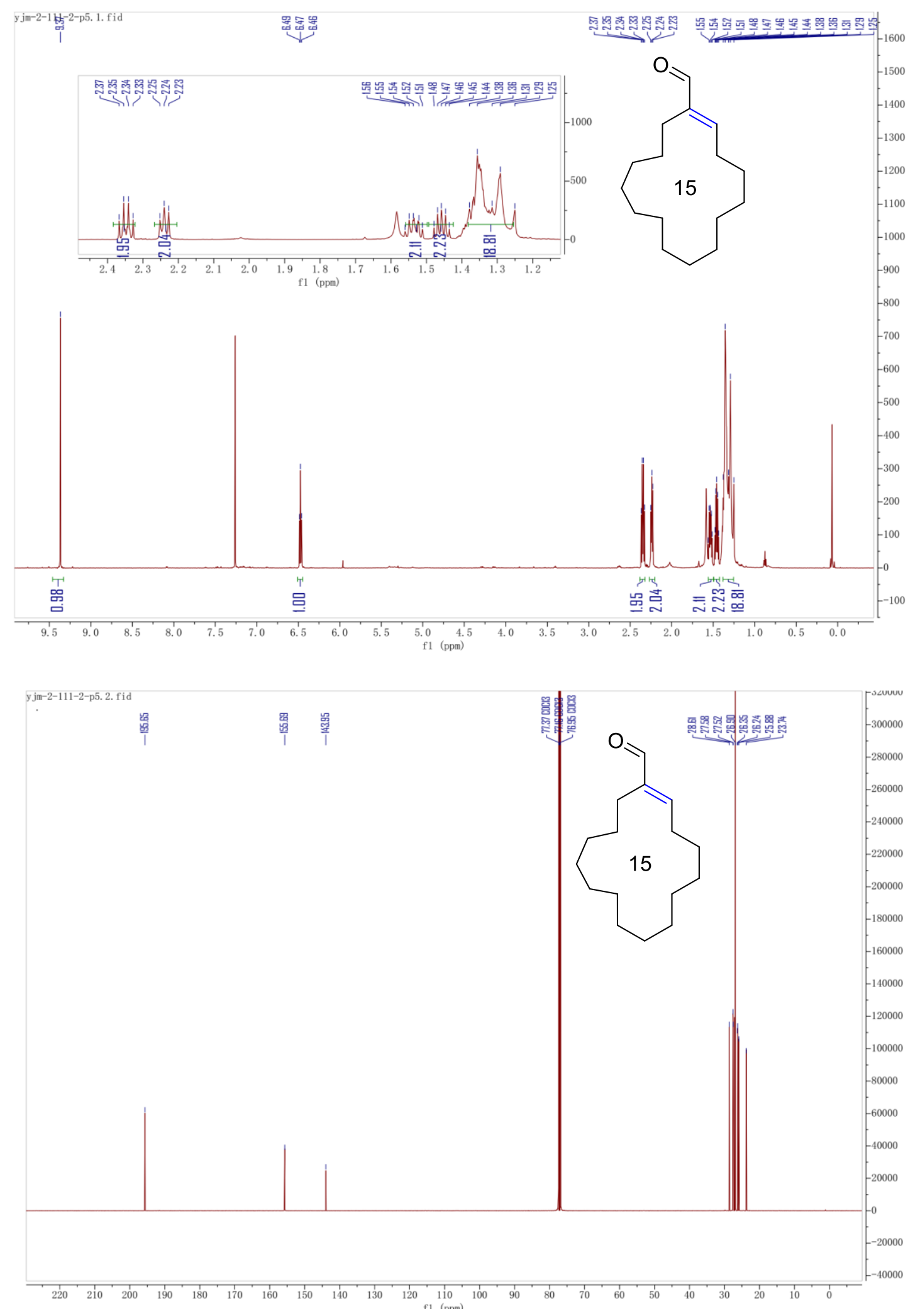
(E)-cycloheptadec-1-ene-1-carbaldehyde $\mathbf{3 f}$
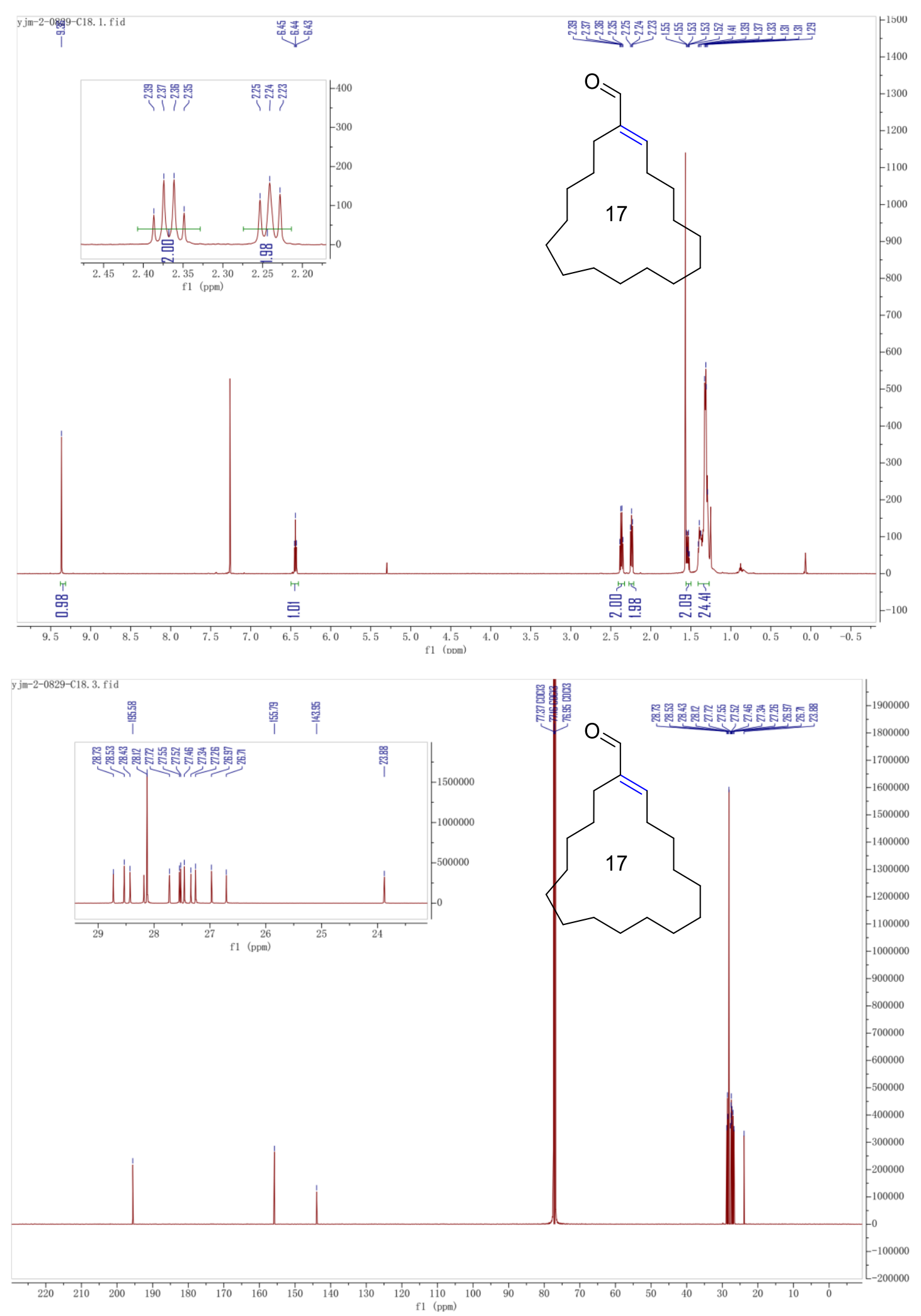
(E)-oxacyclododec-6-ene-6-carbaldehyde 3g
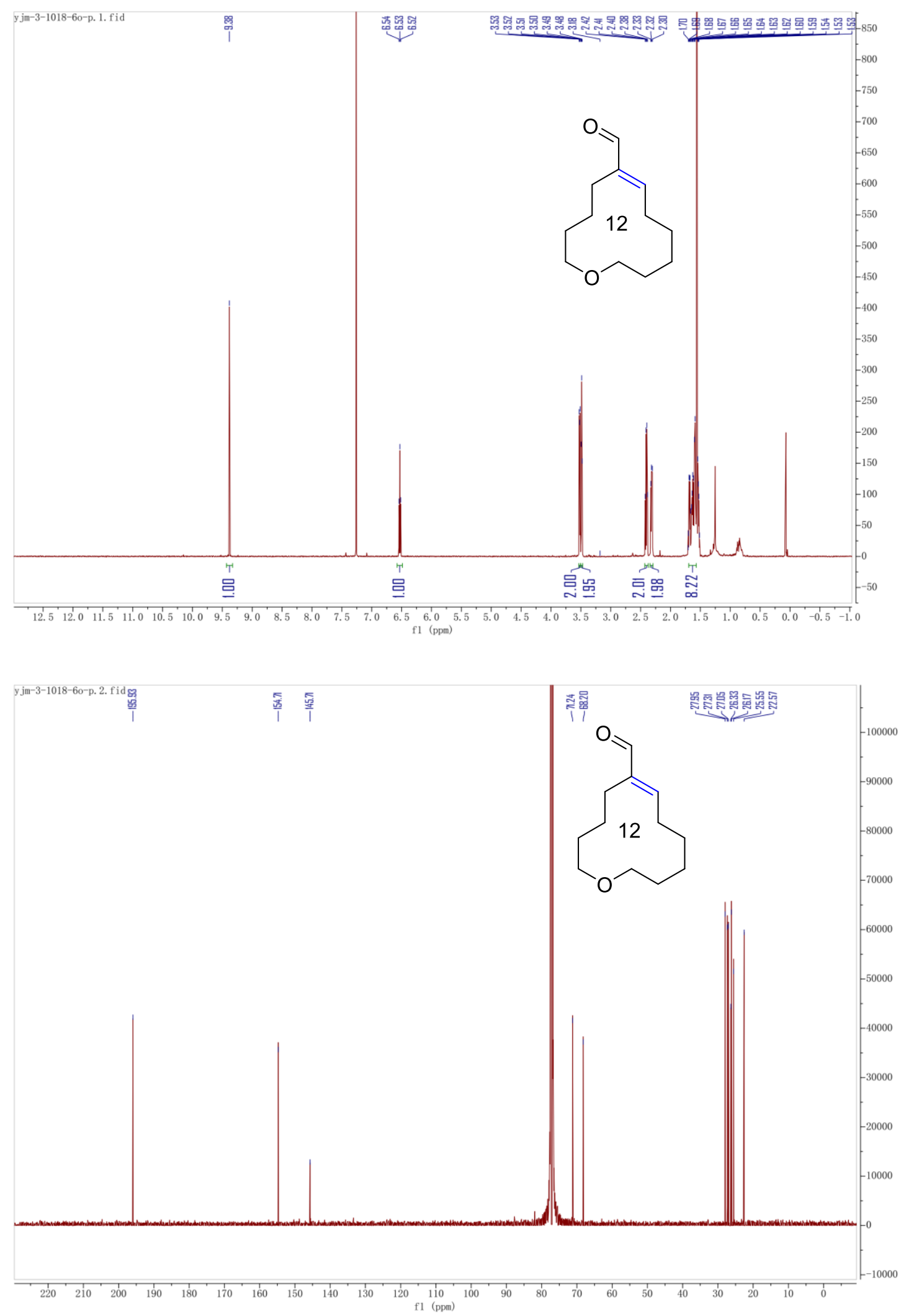
(E)-thiacyclododec-6-ene-6-carbaldehyde $\mathbf{3 h}$

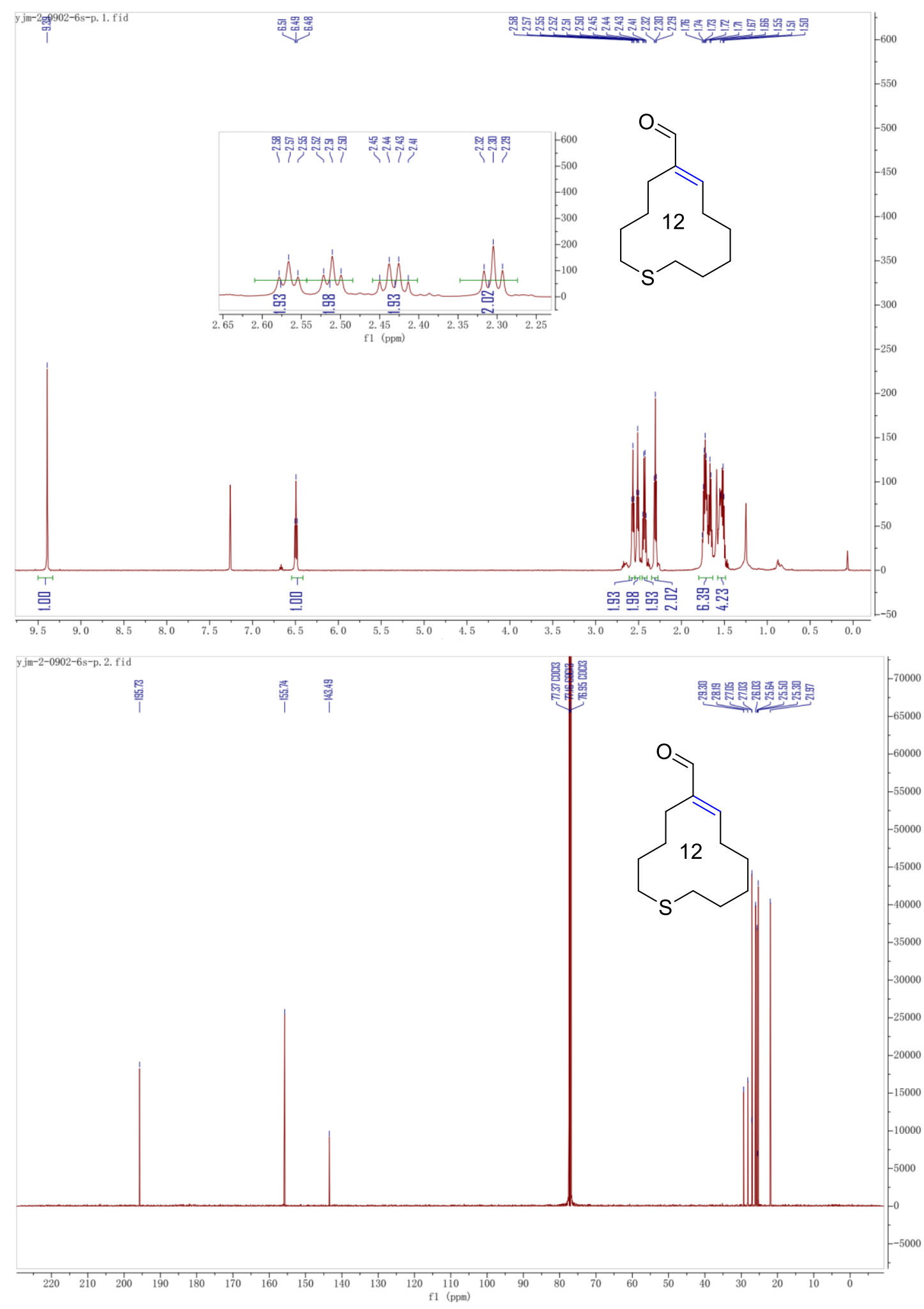


(E)-oxacyclohexadec-8-ene-8-carbaldehyde 3i
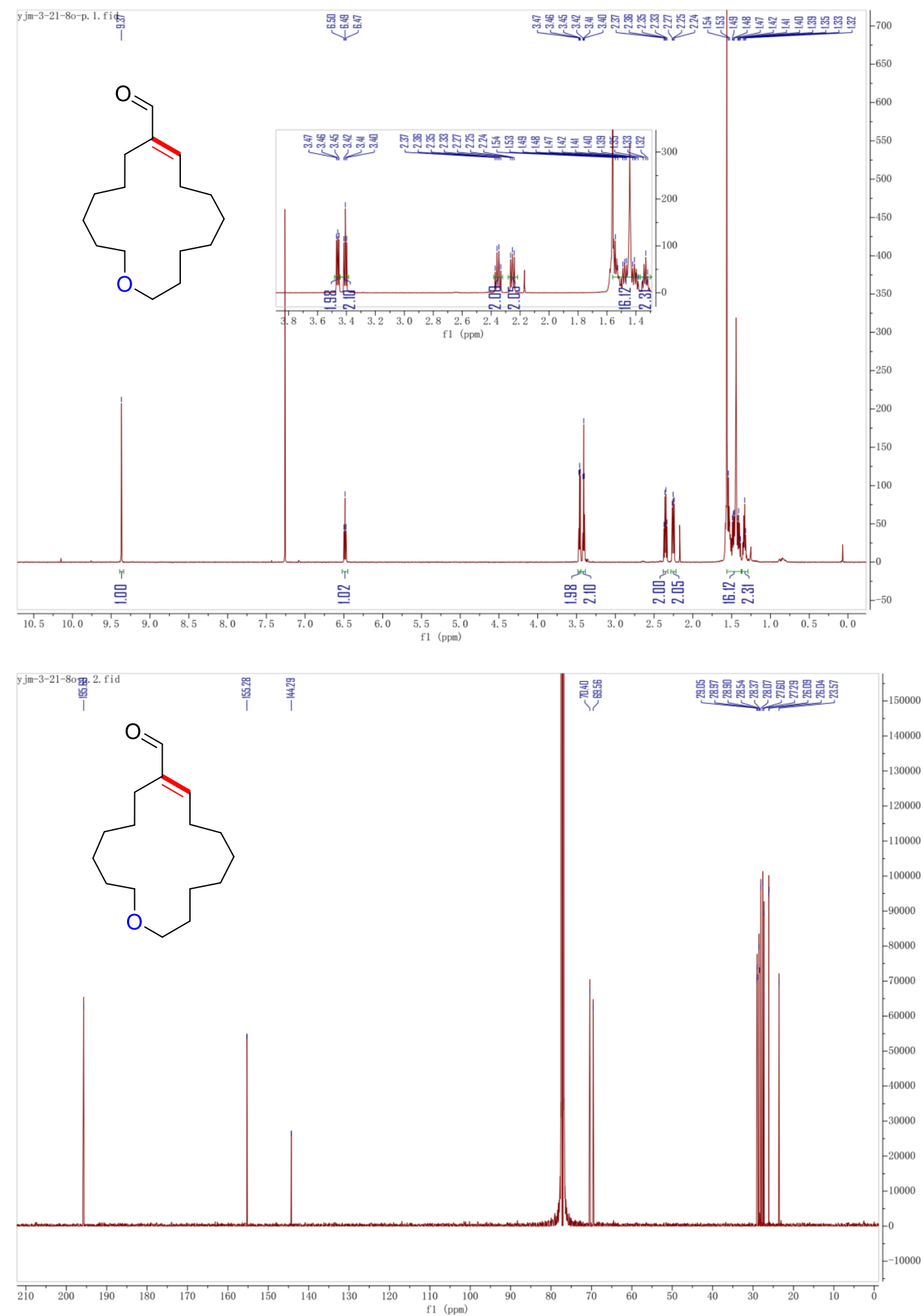
(E)-thiacyclohexadec-8-ene-8-carbaldehyde 3j
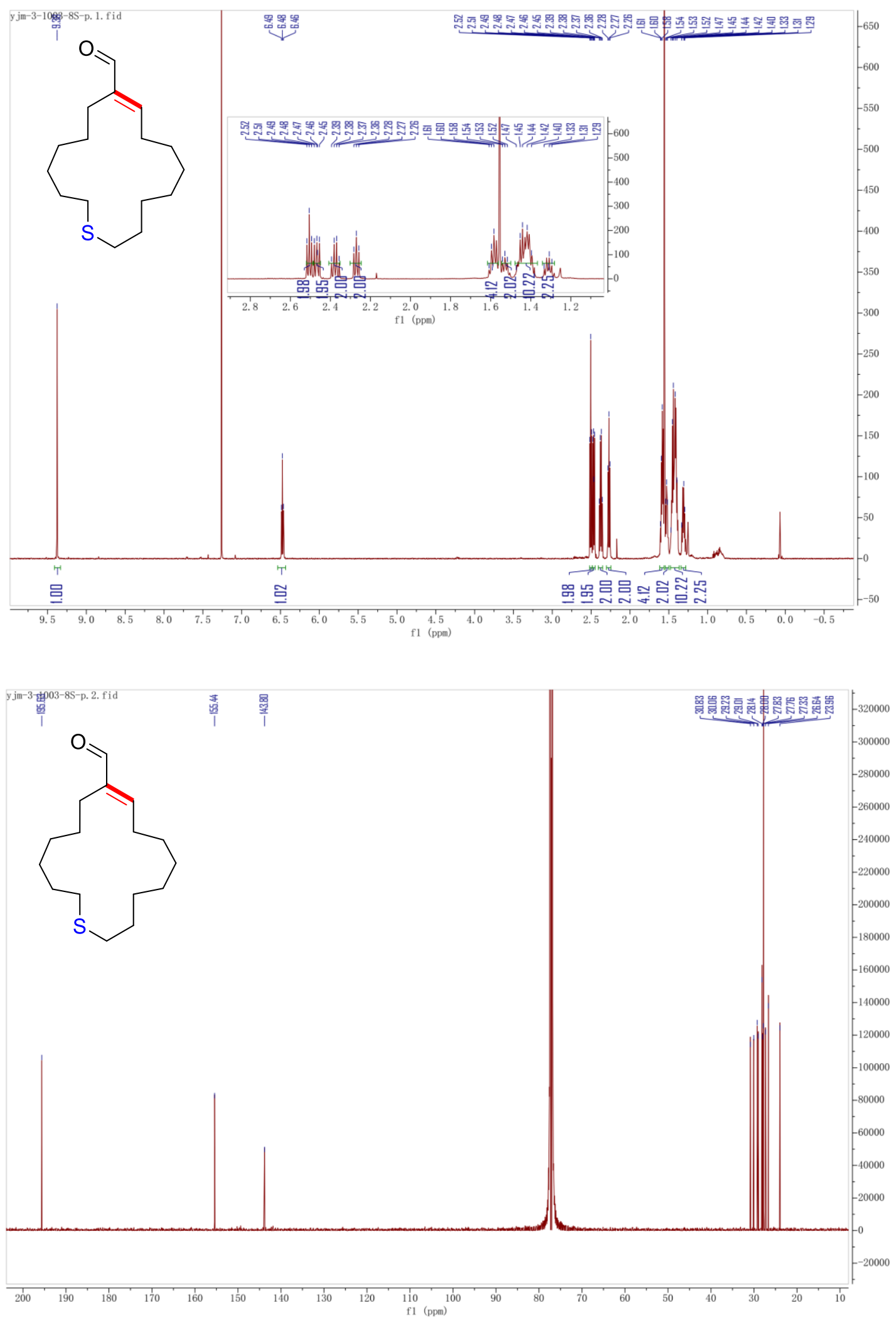


\section{References}

1 Wu, N.-W.; Petsalakis, I. D.; Theodorakopoulos, G.; Yu, Y.; Rebek, J., Jr. Angew. Chem. Int. Ed. 2018, 57, 15091-15095.

2 Roels, J.; Metz P. Synlett 2001,789-790.

3 Suzuki, T.; Tokunaga, M.; Wakatsuki, Y. Org. Lett. 2001, 3, 735-737.

${ }^{4}$ Li, H.; C. L.; Castro, M.; Zheng, J.; Roisnel, T.; Dorcet, V.; Sortais, J.; Darcel, C. Angew. Chem. Int. Ed. 2013, 52, 8045-8049.

5 Ziffle, V. E.; Cheng, P.; Clive, D. L. J. J. Org. Chem. 2010, 75, 8024-8038

${ }^{6}$ Mangaleswaran, S.; Argade, N. P. J. Org. Chem. 2001, 66, 5259-5261.

7 Ganesh, N. V.; Fujikawa, K.; Tan, Y. H.; Nigudkar, S. S.; Stine, K. J.; Demchenko, A. V. J. Org. Chem. 2013, 78, 6849-6857.

8 Tan, G.; Wu, Y.; Shi, Y.; You, J. Angew. Chem. Int. Ed. 2019, 58, 7440-7444. 